OPEN ACCESS

Edited by:

Alan Jay Katz,

St. Francis Hospital, United States

Reviewed by:

Thomas Zilli,

Université de Genève, Switzerland

Raphael Pfeffer,

Assuta Medical Center, Israel

Nuradh Joseph,

Governement of Sri Lanka, Sri Lanka

*Correspondence: Jim Zhong jim.zhong@nhs.net

Specialty section:

This article was submitted to

Radiation Oncology,

a section of the journal

Frontiers in Oncology

Received: 16 March 2021

Accepted: 16 August 2021

Published: 09 September 2021

Citation:

Zhong J, Slevin F, Scarsbrook AF, Serra M, Choudhury A, Hoskin PJ, Brown S and Henry AM (2021)

Salvage Reirradiation Options for Locally Recurrent Prostate Cancer: A Systematic Review.

Front. Oncol. 11:681448. doi: 10.3389/fonc.2021.681448

\section{Salvage Reirradiation Options for Locally Recurrent Prostate Cancer: A Systematic Review}

\author{
Jim Zhong 1,2,3*, Finbar Slevin 2,3, Andrew F. Scarsbrook ${ }^{1,2}$, Maria Serra ${ }^{4}$, \\ Ananya Choudhury ${ }^{4,5}$, Peter J. Hoskin ${ }^{4,5,6}$, Sarah Brown ${ }^{7}$ and Ann M. Henry ${ }^{2,3}$ \\ 1 Department of Diagnostic and Interventional Radiology, Leeds Cancer Centre, St James's University Hospital, Leeds Teaching \\ Hospitals NHS Trust, Leeds, United Kingdom, ${ }^{2}$ Leeds Institute of Medical Research, University of Leeds, Leeds, United Kingdom, \\ ${ }^{3}$ Department of Clinical Oncology, Leeds Cancer Centre, St James's University Hospital, Leeds Teaching Hospitals NHS Trust, \\ Leeds, United Kingdom, ${ }^{4}$ Department of Clinical Oncology, The Christie Hospital, Manchester, United Kingdom, ${ }^{5}$ The Institute of \\ Cancer Sciences, University of Manchester, Manchester, United Kingdom, ${ }^{6}$ Department of Clinical Oncology, Mount Vernon \\ Cancer Centre, Northwood, United Kingdom, ${ }^{7}$ Clinical Trials Research Unit, University of Leeds, Leeds, United Kingdom
}

Background: Reirradiation using brachytherapy (BT) and external beam radiation therapy (EBRT) are salvage strategies with locally radiorecurrent prostate cancer. This systematic review describes the oncologic and toxicity outcomes for salvage BT and EBRT [including Stereotactic Body Radiation Therapy (SBRT)].

Methods: An International Prospective Register of Systematic Reviews (PROSPERO) registered (\#211875) study was conducted using Preferred Reporting Items for Systematic reviews and Meta-analyses (PRISMA) guidelines. EMBASE and MEDLINE databases were searched from inception to December 2020. For BT, both low dose rate (LDR) and high dose rate (HDR) BT techniques were included. Two authors independently assessed study quality using the 18-item Modified Delphi technique.

Results: A total of 39 eligible studies comprising 1967 patients were included (28 BT and 11 SBRT). In 35 studies (90\%), the design was single centre and/or retrospective and no randomised prospective studies were found. Twelve BT studies used LDR only, 11 HDR only, 4 LDR or HDR and 1 pulsed-dose rate only. All EBRT studies used SBRT exclusively, four with Cyberknife alone and 7 using both Cyberknife and conventional linear accelerator treatments. Median (range) modified Delphi quality score was 15 (6-18). Median (range) follow-up was 47.5 months (13-108) (BT) and 25.4 months (21-44) (SBRT). For the LDR-BT studies, the median (range) 2-year and 5-year bRFS rates were 71\% (48-89.5) and 52.5\% (20-79). For the HDR-BT studies, the median (range) 2-year and 5-year bRFS rates were $74 \%$ (63-89) and 51\% (45-65). For the SBRT studies, the median (range) 2-year bRFS for the SBRT group was 54.9\% (40-80). Mean (range) acute and late grade $\geq 3 \mathrm{GU}$ toxicity rates for LDR-BT/HDR-BT/SBRT were 7.4\%(0-14)/2\%(0-14)/2.7\%(0-8.7) and 13.6\%(0-30)/ $7.9 \%(0-21.3 \%) / 2.7 \%(0-8 \%)$. Mean (range) acute and late grade $\geq 3 \mathrm{Gl}$ toxicity rates for LDR-BT/HDR-BT/SBRT were 6.5\%(0-19)/0\%/0.5\%(0-4\%) and 6.4\%(0-20)/0.1\%(0-0.9)/ $0.2 \%(0-1.5)$. One third of studies included Patient Reported Outcome Measures (PROMs).

Conclusions: Salvage reirradiation of radiorecurrent prostate cancer using HDR-BT or SBRT provides similar biochemical control and acceptable late toxicity. Salvage LDR-BT 
is associated with higher late GU/GI toxicity. Challenges exist in comparing BT and SBRT from inconsistencies in reporting with missing data, and prospective randomised trials are needed.

Keywords: prostate cancer, local recurrence, reirradiation, salvage, brachytherapy, external beam radiotherapy (EBRT), stereotactic body radiotherapy (SBRT)

\section{INTRODUCTION}

Prostate cancer is the most common male cancer accounting for over 1.2 million new cases per year with $>350,000$ deaths $(3.8 \%$ of all male cancer deaths) (1). Radiation therapy (RT) is a curative treatment option for localised prostate cancer and can be offered to patients from all risk groups (2). Despite advances in diagnostic imaging, RT delivery techniques and dose-escalated radiation, biochemical progression remains common and occurs in $15-57 \%$ of patients with localised disease (3-5).

Multiple salvage options are available for locally recurrent non-metastatic disease including prostatectomy, reirradiation [with brachytherapy (BT) or external beam radiotherapy (EBRT)] and other focal therapies such as high-intensity focused ultrasound (HIFU) and cryotherapy. However, there is limited evidence to support the effectiveness of salvage therapies with concerns regarding the potential for significant toxicity that may impact the long-term quality of life of patients. Due to uncertainty regarding benefits and risks of harm only $15-20 \%$ of patients with locally recurrent prostate cancer undergo salvage therapy according to the Cancer of the Prostate Strategic Urologic Research Endeavor (CaPSURE) registry (6).

BT has been preferred for reirradiation as it offers delivery of highly conformal high dose radiation with a steep dose gradient and rapid fall off which minimises dose to surrounding organs at risk (7). Disadvantages of BT include its invasive nature and the need for a specialist multi-disciplinary team not available in all radiation centres. Previously, EBRT techniques have been associated with high rates of severe late toxicities and poor local control (8). Stereotactic body radiation therapy (SBRT) involves delivery of a high dose of external beam radiation to a highly conformal target volume with a steep dose gradient in a small number of fractions and is now under investigation for locally recurrent prostate cancer. Advantages of this approach are that it is non-invasive and has the potential to be delivered in more radiation centres than $\mathrm{BT}(9)$.

This systematic review collates the most up-to-date evidence for reirradiation of locally recurrent prostate cancer. Two previous systematic reviews which compared all salvage therapies found higher biochemical control rates for BT and EBRT compared to surgical and other non-surgical local therapies [high intensity focused ultrasound (HIFU) and cryotherapy] along with potentially lower genitourinary (GU) toxicity $(10,11)$. The justification for this systematic review is that the identification of the reirradiation modality that offers optimum prostate cancer control and minimal toxicity is important to enable patients to make informed decisions and potentially improve outcomes in patients with radiorecurrent prostate cancer. In addition, the evidence base for salvage BT and SBRT continues to expand with a number of new publications in the past 1-2 years.

\section{METHODS AND MATERIALS}

An International Prospective Register of Systematic Reviews registered (\#211875) systematic review was conducted.

\section{Study Design}

The study followed the Preferred Reporting Items for Systematic Reviews and Meta-analysis (PRISMA) guidelines (12).

Studies were identified by searching the Cochrane library, EMBASE and MEDLINE electronic databases from inception to $14^{\text {th }}$ December 2020.

The search strategy is documented in Supplementary Material 1 and the combination of subject headings and keywords included: 'recurrent or radiorecurrent prostate cancer', 'reirradiation' or 'reirradiation', 'salvage radiotherapy', 'brachytherapy', 'external beam radiotherapy', 'stereotactic body radiation therapy', 'stereotactic ablative radiotherapy', 'radiosurgery'.

\section{Data Extraction}

Two authors (JZ and FS) independently reviewed the abstracts and assessed the quality of each study using an 18-item Modified Delphi technique, which has been previously validated for case series (13). Discordance between reviewers were resolved following arbitration by a third reviewer $(\mathrm{AH})$.

\section{Data Selection}

Eligible studies included patients treated with primary EBRT, BT or combination EBRT/BT and salvage therapy for local recurrence with either EBRT or BT. For BT techniques, studies of high-dose rate brachytherapy (HDR-BT), low-dose rate brachytherapy (LDR-BT) and pulse-dose rate brachytherapy (PDR-BT) were included.

Studies that predominantly included patients who had primary treatment with radical prostatectomy, cryotherapy or HIFU were not included in this review as the focus was to collate and present the most up-to-date evidence concerning reirradiation specifically.

Studies with fewer than 20 patients were excluded, along with editorials, letters, abstracts, case reports, conference proceedings and studies not written in English. Where studies had evaluated the same patient cohort as another publication, only the most recent publication was used for analysis unless distinct treatment outcomes or toxicity were described. 


\section{EXTRACTED VARIABLES}

Extracted data included the first author and country in which the study took place, study type (prospective or retrospective), single/ multi-centre status, number of patients, primary disease characteristics, primary treatment modalities, interval between original treatment and salvage treatment, patient age at salvage, pre-salvage prostate specific antigen (PSA), diagnostic imaging prior to salvage treatment, histological confirmation of local recurrence and percentage of biopsy-proven recurrences in study cohort, whole-gland salvage treatment versus focal salvage treatment, type of salvage radiotherapy (HDR-BT, LDR-BT, PDRBT or EBRT), salvage dose fractionation schedule, percent of patients who received androgen deprivation therapy (ADT) with their salvage treatment, duration of follow up post salvage therapy, treatment outcomes [biochemical control (BC), biochemical recurrence free survival (bRFS), metastasis free survival (MFS), relapse free survival (RFS), cancer specific survival (CSS), overall survival (OS)] and grade 1-4 GU and gastrointestinal (GI) toxicity as classified by the Common Terminology Criteria for Adverse Events (CTCAE) (14) or Radiation Therapy Oncology Group (RTOG) score (15). Use of any patient recorded outcome measure (PROM) in the study was also collated including the type of tool used. Median (range) values calculated for all collected variables except toxicity rates where mean (range) used to account for the studies which report no toxicity.

\section{RESULTS}

From the initial identification of 2744 articles, a total of 39 studies were included in the final analysis. A PRISMA flowchart of the systematic review is presented in Figure 1. The last electronic literature search was performed on $14^{\text {th }}$ December 2020.

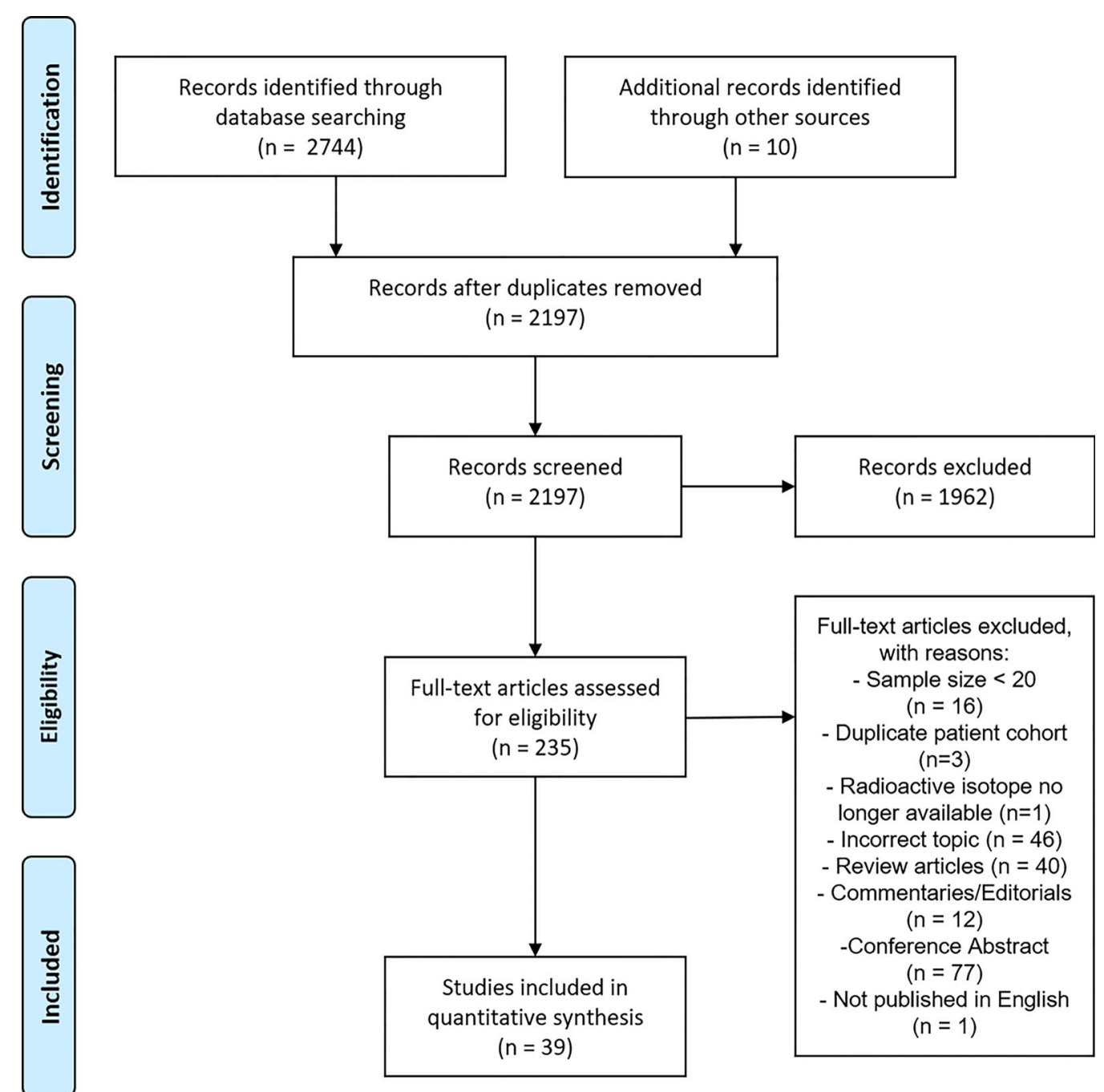

FIGURE 1 | PRISMA flow chart of literature search. 
The quality assessment tool (modified Delphi 18-item checklist) scores for all included studies are shown in Supplementary Table 1 . The summary of these results is shown in Table 1. The median modified Delphi score was 15 out of 18 (83.3\%) (range 6-18).

\section{Treatment Details}

A summary of patient, disease and treatment characteristics at the time of primary treatment and salvage treatments for BT and EBRT studies is shown in Tables $\mathbf{2 - 5}$ respectively. Salvage treatment characteristics for BT and EBRT are shown in Tables 6, 7 respectively.

Twenty-eight BT studies were included with a total of 1484 patients treated: 22 were retrospective and 6 were prospective. Four were multi-centre and 24 were single centre. Twelve BT studies used LDR only (16-27), 11 used HDR only (28-39), 4 used LDR or HDR (40-43) and 1 used PDR only (44). Twenty four studies used whole gland salvage treatments and 4 studies used focal salvage treatments $(16,34,36,38)$. The number of HDR-BT fractions ranged from 1 to 4 (median of 3 fractions) and the inter-fraction time interval ranged from 4 hours to 3 weeks. The median overall salvage treatment time was 21 days (range 1 to 63 days).

All EBRT studies $(n=11)$ used an SBRT technique with a total of 483 patients treated. Of these studies $(9,45-54), 9$ were retrospective and 2 were prospective $(46,47)$. Two were multicentre and 9 were single centre. Four studies used Cyberknife delivery only and 7 studies included patients treated with
Cyberknife or conventional linear accelerator SBRT techniques. Four studies used whole gland salvage only, 4 focal salvage only, 2 included both whole gland and focal treatments and one did not specify. Of the 11 studies, 8 were published between 2019 and 2020. The median total radiation dose prescribed was $34 \mathrm{~Gy}$ (range 34-38 Gy), over a median of 5 fractions (range 3-7). The median overall treatment time was 6 days (range 3-14 days).

The median number (range) of included patients for individual BT and SBRT studies was 44 (21-115) and 42 (23$100)$ respectively. The median age (range) at salvage treatment was 70 years (59-76) for BT studies and 74 years (64-78) for SBRT studies. The median PSA at primary treatment for the BT and SBRT studies were $10.9 \mathrm{ng} / \mathrm{mL}$ (range 7.4-26.4) and $10.3 \mathrm{ng} /$ $\mathrm{mL}$ (range 8.7-13.0) respectively. The median PSA at salvage treatment for the BT and SBRT studies were $4.7 \mathrm{ng} / \mathrm{mL}$ (range 2.8-11.4) and $3.1 \mathrm{ng} / \mathrm{mL}$ (range 2.5-4.1) respectively. The median time from primary treatment to salvage therapy for the BT and SBRT studies were 67 months (range 30-101 months) and 86.5 months (range 60-100 months) respectively.

Seventeen studies (44\%) used both multi-parametric magnetic resonance imaging (mpMRI) and positron emission tomography-computed tomography (PET-CT) for restaging prior to salvage treatment. Four studies (10\%) used prostatespecific membrane antigen (PSMA) PET-CT and 13 studies (33\%) used choline/fluciclovine PET-CT for re-staging. Eight studies (all BT) (21\%) used computed tomography (CT) or isotope bone scintigraphy for restaging. Ten studies $(26 \%)$ did not report the imaging modality used for restaging. Among the

TABLE 1 | Summary findings from the Modified Delphi checklist for quality assessment applied to all included studies ( $n=39$ ).

Criterion

\begin{tabular}{ll}
\multicolumn{3}{c}{ Studies, $\mathbf{n}(\%)$} \\
\hline Yes & No
\end{tabular}

\section{Study Objective}

1. Is the hypothesis/aim/objective of the study clearly stated in the abstract, introduction, or methods section? Study population

$8(97.4)$

2. Are the characteristics of the participants included in the study described?

3. Were the cases collected in more than 1 Centre?

4. Are the eligibility criteria (inclusion and exclusion criteria) to entry the study explicit and appropriate?

5. Were the participants recruited consecutively?

6. Did participants enter the study at a similar point in the disease?

9. Are the outcome measures clearly defined in the introduction or methods section?

10. Were relevant outcomes appropriately measured with objective/or subjective methods?

$100(100)$

$8(20.5)$

33 (84.6)

$26(66.7)$

35 (89.7)

37 (94.9)

35 (89.7)

11. Were outcomes measured before and after intervention?

$35(89.7)$

Statistical analysis

12. Were the statistical tests used to assess the relevant outcomes appropriate?

Results and conclusions

13. Was the length of follow-up reported?

14. Was the loss of follow-up reported?

15. Does the study provide estimates of the random variability in the data analysis of relevant outcomes?

16. Are adverse events reported?

17. Are the conclusions of the study supported by results?

Competing interest and source of support

18. Are both competing interest and source of support for the study reported?

Median Modified Delphi score = 15 out of 18 (83.3\%) (range 6-18)
38 (97.4)

38 (97.4)

$23(59.0)$

$15(38.5)$

38 (97.4)

38 (97.4)

23 (59.0)
4 (10.3)

2 (5.1)

4 (10.3) 
TABLE 2 | Primary disease and treatment characteristics for brachytherapy studies

\begin{tabular}{|c|c|c|c|c|c|c|c|c|c|c|c|c|c|c|}
\hline $\begin{array}{l}\text { First author } \\
\text { (country) }\end{array}$ & Year & $\begin{array}{l}\text { Salvage BT } \\
\text { type }\end{array}$ & Design & $\begin{array}{l}\text { Pts } \\
(n)\end{array}$ & $\begin{array}{l}\text { PSA (ng/mL) } \\
\text { (range) }\end{array}$ & ISUP & GS & $\begin{array}{c}\% \text { GS } \\
(\leq 7)\end{array}$ & $\begin{array}{c}\% \text { GS } \\
(\geq 8)\end{array}$ & $\begin{array}{c}\mathrm{T} \\
\text { stage }\end{array}$ & 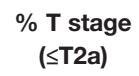 & $\begin{array}{c}\% \text { T stage } \\
\text { ( } \geq \mathrm{T} 2 \mathrm{~b})\end{array}$ & Risk Class & Primary treatment \\
\hline B Lee (USA) & 2007 & HDR & $\mathrm{R}$ & 21 & NR & 1 & 6 & 100 & 0 & T2c & 48 & 52 & NR & EBRT, BT, protonTx \\
\hline Lyczek (Poland) & 2009 & HDR & R & 115 & $13(2.34-64.5)$ & NR & NR & NR & NR & T2 & 58 & 42 & NR & $\begin{array}{l}\mathrm{RP}+\mathrm{EBRT}, \mathrm{EBRT}, \mathrm{BT} \\
\mathrm{EBRT}+\mathrm{BT}\end{array}$ \\
\hline Chen (USA) & 2013 & HDR & $\mathrm{R}$ & 52 & $9.3(1.2-58)$ & 1 & 6 & 87 & 13 & T2 & NR & NR & NR & EBRT, BT, EBRT+BT, PBT \\
\hline Kukielka (Poland) & 2014 & HDR & $\mathrm{R}$ & 25 & $16.3(6.37-64)$ & 1 & $<6$ & 88 & 4 & T2c & 48 & 52 & Intermediate & EBRT \\
\hline *Yamada (USA) & 2014 & $\mathrm{HDR}$ & $P$ & 42 & NR & NR & NR & NR & NR & NR & NR & NR & NR & EBRT \\
\hline Jiang (Germany) & 2016 & HDR & $R$ & 29 & $N R$ & $N R$ & $N R$ & $N R$ & $N R$ & $N R$ & $N R$ & NR & High & EBRT, EBRT+BT \\
\hline Lacy (USA) & 2016 & HDR & $\mathrm{R}$ & 21 & NR & $N R$ & $N R$ & NR & $N R$ & NR & $N R$ & NR & Low & $\mathrm{BT}, \mathrm{EBRT}+\mathrm{BT}$ \\
\hline Wojcieszek (Poland) & 2016 & HDR & $\mathrm{R}$ & 83 & 13.7 & 1 & 6 & 80 & 4 & $\mathrm{~T} 2$ & $N R$ & NR & Intermediate & EBRT, EBRT+BT \\
\hline \multirow[t]{2}{*}{ Lopez (Spain) } & 2019 & HDR & R & 75 & $8.9(3.5-42.1)$ & 1 & 6 & 75 & 20 & NR & $N R$ & NR & Intermediate & EBRT, BT \\
\hline & 2019 & LDR & $\mathrm{R}$ & 44 & $14.2(3.2-167)$ & 1 & 6 & 87 & 11 & NR & NR & NR & Intermediate & EBRT \\
\hline Chitmanee (UK) & 2020 & HDR & $P$ & 50 & $<10(46 \%)$ & $2 / 3$ & 7 & 90 & 10 & $\mathrm{~T} 2$ & 72 & 28 & Intermediate & EBRT, BT \\
\hline Slevin (UK) & 2020 & HDR & $\mathrm{R}$ & 43 & $10.5(3.4-178)$ & 1 & 6 & 90 & 10 & T2 & $73 \%$ & $27 \%$ & Intermediate & EBRT, BT \\
\hline $\begin{array}{l}\text { van Son } \\
\text { (Netherlands) }\end{array}$ & 2020 & HDR & $P$ & 50 & $13(2.1-140)$ & 1 & 6 & 82 & 12 & T2a & 72 & 28 & NR & EBRT, BT \\
\hline Kollmeier (USA) & 2017 & HDR/LDR & $\mathrm{R}$ & 98 & $\leq 10(74 \%)$ & 2 & 7 & 92 & 8 & $\mathrm{~T} 2 \mathrm{~b}$ & 68 & 32 & NR & EBRT, BT, EBRT+BT \\
\hline Baumann (USA) & 2017 & HDR/LDR & $\mathrm{R}$ & 33 & $8.4(3.8-68.7)$ & NR & 7 & 79 & 21 & $\mathrm{~T} 2$ & 55 & 45 & High & EBRT \\
\hline Henriquez (Spain) & 2014 & HDR/LDR & $R$ & 56 & $10.7(4-121)$ & 1 & 6 & 95 & 5 & $\mathrm{~T} 2$ & 87 & 13 & Intermediate & EBRT, BT \\
\hline Grado (USA) & 1999 & LDR & $\mathrm{R}$ & 49 & $26.4(2.3-95.8)$ & $N R$ & $\begin{array}{c}7 \text { to } \\
10\end{array}$ & $N R$ & $N R$ & $\mathrm{~T} 2 \mathrm{~b}$ & NR & $N R$ & $N R$ & EBRT, BT, RP \\
\hline Koutrouvelis (USA) & 2003 & LDR & R & 31 & $<10(32 \%)$ & NR & 6 & 77 & 23 & $\begin{array}{l}\mathrm{T} 2 \mathrm{~b} / \\
\mathrm{T} 3 \mathrm{a}\end{array}$ & $32 \%$ & $68 \%$ & NR & BT \\
\hline Nguyen (USA) & 2007 & LDR & $\mathrm{P}$ & 25 & $7.4(4.2-18.4)$ & 1 & 6 & 100 & 0 & $\mathrm{~T} 1 \mathrm{c}$ & NR & NR & NR & EBRT, EBRT+BT \\
\hline HK Lee (USA) & 2008 & LDR & $\mathrm{R}$ & 21 & NR & $N R$ & $N R$ & NR & $N R$ & NR & NR & NR & NR & EBRT \\
\hline Aaronson (USA) & 2009 & LDR & $\mathrm{R}$ & 24 & $9.9(3.2-69)$ & 3 & 7 & 71 & 12 & T1c & NR & NR & NR & EBRT \\
\hline Burri (USA) & 2010 & LDR & $\mathrm{R}$ & 37 & $10.9(4.4-81)$ & NR & 6 & 73 & 11 & NR & 19 & 16 & Intermediate & EBRT, BT \\
\hline $\begin{array}{l}\text { Moman } \\
\text { (Netherlands) }\end{array}$ & 2010 & LDR & R & 31 & 24.3 & NR & 7 & 84 & 6.5 & $\mathrm{~T} 2$ & NR & NR & $\mathrm{NR}$ & EBRT, BT \\
\hline Peters (Netherlands) & 2014 & LDR & $\mathrm{R}$ & 20 & $12.9(5.4-51)$ & 1 & 6 & 90 & 10 & T3 & NR & NR & High & EBRT, BT \\
\hline Vargas (USA) & 2014 & LDR & $\mathrm{R}$ & 69 & $<10(62 \%)$ & 1 & 6 & 80.3 & 19.7 & T2 & NR & NR & NR & EBRT \\
\hline Peters (Netherlands) & 2016 & LDR & R & 62 & $16.6(2.6-66.9)$ & $2 / 3$ & 7 & 95 & 5 & T2 & 66 & 34 & NR & EBRT, BT \\
\hline Crook (Canada) & 2019 & LDR & $P$ & 92 & NR & NR & 7 & 100 & 0 & NR & NR & NR & $\begin{array}{l}\text { low/ } \\
\text { intermediate }\end{array}$ & EBRT \\
\hline Smith (USA) & 2020 & LDR & $P$ & 108 & $9.15(1.7-116)$ & 1 & 6 & 54 & 10 & $\mathrm{~T} 2$ & 67 & 5 & Intermediate & EBRT \\
\hline Schonle (Germany) & 2020 & PDR & $\mathrm{R}$ & 82 & $9(0.9-170)$ & $2 / 3$ & 7 & 74 & 11 & NR & NR & NR & Intermediate & EBRT, BT, RP \\
\hline
\end{tabular}

BT, brachytherapy; HDR, high dose rate; LDR, low dose rate; $R$, retrospective; $P$, prospective; Pts, patients, $n$, nnumber; PSA, prostate specific antigen; NR, not recorded; GS, Gleason score; EBRT, external beam radiotherapy; PBT, proton beam treatment; $R P$, radical prostatectomy.

For PSA, ISUP and GS, the median scores are presented.

*Yamada (USA) study cohort included in further publication Kollmeier (USA) however specific treatment characteristics and toxicity were not covered in later paper. 
TABLE 3 | Pre-salvage therapy disease and treatment characteristics for brachytherapy studies.

\begin{tabular}{|c|c|c|c|c|c|c|c|c|c|c|c|c|}
\hline First author (country) & Year & Salvage BT type & TRS (mo)(range) & BCR definition & Age (years)(range) & PSA (ng/mL)(range) & ISUP & GS & $\%$ GS $(\leq 7)$ & $\%$ GS ( $\geq 8)$ & Imaging for relapse & Biopsy \\
\hline B Lee (USA) & 2007 & HDR & $63.6(24-125)$ & NR & $68(58-81)$ & $5.9(1.4-9.5)$ & NR & NR & 52 & 38 & MRI & Yes (100\%) \\
\hline Lyczek (Poland) & 2009 & HDR & 49.5 & NR & $70(52-82)$ & NR & 1 & 6 & 71 & 12 & NR & No \\
\hline Chen (USA) & 2013 & HDR & $51.6(10.8-135.6$ & Phoenix & $67.5(53.9-81.4)$ & $5(0.4-26.3)$ & NR & 8 & 48 & 52 & CT & Yes (100\%) \\
\hline Kukielka (Poland) & 2014 & HDR & NR & Phoenix & $71(62-83)$ & $2.8(1.04-25.3)$ & $2 / 3$ & 7 & 60 & 20 & MRI & Yes (100\%) \\
\hline *Yamada (USA) & 2014 & HDR & 73 & Phoenix & 72 & 3.54 & $N R$ & 7 & 67 & 33 & MRI, BS & Yes (100\%) \\
\hline Jiang (Germany) & 2016 & HDR & NR & Phoenix & $75.5( \pm 5.8)$ & $4.05(2.1-18.6)$ & NR & NR & NR & NR & C-PET & No \\
\hline Lacy (USA) & 2016 & HDR & $45(4-287)$ & Phoenix & $59(44-72)$ & $6.3(1-19.1)$ & NR & NR & NR & NR & $\mathrm{CT}, \mathrm{BS}$ & Yes (14\%) \\
\hline Wojcieszek (Poland) & 2016 & HDR & $67(22-124)$ & NR & $70(57-81)$ & $3.1(0.1-19.9)$ & $\mathrm{NR}$ & 7 & 46 & 7 & MRI, BS & Yes (100\%) \\
\hline \multirow[t]{2}{*}{ Lopez (Spain) } & 2019 & HDR & $>30$ & ASTRO/ Phoenix & $62.1(4-75)$ & $4.1(1.5-16.7)$ & NR & 8 to 10 & 48 & 44 & $\begin{array}{l}\text { CT, MRI, } \\
\text { C-PET }\end{array}$ & Yes (100\%) \\
\hline & 2019 & LDR & $>30$ & ASTRO/ Phoenix & $60.4(47-71)$ & $3.6(1.02-11)$ & $2 / 3$ & 7 & 59 & 9 & $\begin{array}{l}\text { CT, MRI, } \\
\text { C-PET }\end{array}$ & Yes (100\%) \\
\hline Chitmanee (UK) & 2020 & HDR & $<5$ years & Phoenix & $70(57-82)$ & $<10(94 \%)$ & $2 / 3$ & 7 & 54 & 36 & MRI, C-PET & Yes (100\%) \\
\hline Slevin (UK) & 2020 & HDR & 70 & Phoenix & $70(62-81)$ & $3.1(1.1-7.5)$ & 2 & 7 & 70 & 30 & MRI, PET & Yes (100\%) \\
\hline van Son (Netherlands) & 2020 & HDR & $101(25-228)$ & Phoenix & $71(59-83)$ & $5(0.9-39)$ & 2 & 7 & 74 & 20 & PSMA-PET, MRI & Yes (100\%) \\
\hline Kollmeier (USA) & 2017 & HDR/LDR & $72(12-172)$ & Phoenix & $73.5(56-88)$ & $3.7(0-59)$ & 2 & 7 & 61 & 39 & CT, MRI, BS & Yes (100\%) \\
\hline Baumann (USA) & 2017 & HDR/LDR & $56.1(18-118)$ & Phoenix & $75(57-85)$ & $5(2-26)$ & NR & 7 & 55 & 36 & CT, MRI, BS & Yes (100\%) \\
\hline Henriquez (Spain) & 2014 & HDR/LDR & NR & Phoenix & $65(60-80)$ & $3.7(1.1-30)$ & $2 / 3$ & 7 & 41 & 14 & MRI & Yes (100\%) \\
\hline Grado (USA) & 1999 & LDR & NR & 2 PSA rises $>$ nadir & $73.3(52.9-86.9)$ & $5.6(1.5-79.1)$ & NR & NR & NR & NR & CT & Yes (100\%) \\
\hline Koutrouvelis (USA) & 2003 & LDR & 30 & $\mathrm{nPSA}+1.5$ & $65(51-79)$ & NR & $N R$ & 6 & NR & $N R$ & $N R$ & Yes (100\%) \\
\hline Nguyen (USA) & 2007 & LDR & $62.4(30-153)$ & ASTRO & 65 & $5.5(1.4-11.6)$ & NR & NR & NR & NR & MRI & Yes (100\%) \\
\hline HK Lee (USA) & 2008 & LDR & $85( \pm 30.1)$ & Phoenix & $72( \pm 4.8)$ & 3.8 & $N R$ & 7 & NR & NR & $N R$ & Yes (100\%) \\
\hline Aaronson (USA) & 2009 & LDR & $49(26-109)$ & Phoenix & $66(54-88)$ & $3.41(0.3-10)$ & $N R$ & $N R$ & NR & NR & MRI & Yes (100\%) \\
\hline Burri (USA) & 2010 & LDR & $62(26-171)$ & Phoenix & $70.2(51-79)$ & $5.6(1.7-35)$ & NR & 8 & 65 & 32 & CT & Yes (100\%) \\
\hline Moman (Netherlands) & 2010 & LDR & 60 & ASTRO/ Phoenix & 69.3 & 11.4 & NR & 8 & 70.1 & 12.9 & NR & Yes (100\%) \\
\hline Peters (Netherlands) & 2014 & LDR & $79(42-144)$ & Phoenix & $69(59-78)$ & $4.7(0.3-14)$ & $N R$ & 7 & 65 & 35 & MRI, CT/BS, C-PET & Yes (100\%) \\
\hline Vargas (USA) & 2014 & LDR & 90 & Phoenix & $72.5(55-88)$ & NR & NR & 7 & 73.2 & 26.8 & $\mathrm{CT}, \mathrm{BS}$ & Yes (100\%) \\
\hline Peters (Netherlands) & 2016 & LDR & $67( \pm 32)$ & Phoenix & $69( \pm 5.3)$ & $8.6(0.1-92.6)$ & $N R$ & $N R$ & $N R$ & $N R$ & MRI, PET, BS & Yes (100\%) \\
\hline Crook (Canada) & 2019 & LDR & 85 (39-199) & Phoenix & $70(55-82)$ & $4.1(0.4-9.7)$ & NR & NR & NR & NR & MRI & Yes (100\%) \\
\hline Smith (USA) & 2020 & LDR & $70(10-235)$ & Phoenix & $70(51-87)$ & $5.3(0.1-38.4)$ & 3 & 7 & 65 & 32 & MRI & Yes (100\%) \\
\hline Schonle (Germany) & 2020 & PDR & $87.5(19-255)$ & Phoenix & $69.9(51-83)$ & $5.07(0.28-51)$ & $2 / 3$ & 7 & 59 & 24 & MRI & NR \\
\hline
\end{tabular}

$B T$, brachytherapy; HDR, high dose rate; LDR, low dose rate; PSA, prostate specific antigen; NR, not recorded; GS, Gleason score; TRS, median time from primary treatment to salvage therapy; mo, months; BCR, biochemical recurrence; ASTRO, American Society for Radiation Oncology; MRI, magnetic resonance imaging; NS, bone scan; CT, computed tomography; US, ultrasound; C-PET, Choline positron emission tomography; PSMA, prostate specific membrane antigen. For TRS, age, PSA, ISUP and GS, the median scores are presented.

*Yamada (USA) study cohort included in further publication Kollmeier (USA) however specific treatment characteristics and toxicity were not covered in later paper. 
TABLE 4 | Primary disease and treatment characteristics for EBRT studies

\begin{tabular}{|c|c|c|c|c|c|c|c|c|c|c|c|c|c|}
\hline First author/ country & Year & Design & Pts $(n)$ & PSA (range) (ng/mL) & ISUP & GS & $\%$ GS $(\leq 7)$ & $\%$ GS ( $\geq 8)$ & T stage & $\%$ T stage $(\leq \mathrm{T} 2 \mathrm{a})$ & $\%$ T stage $(\geq T 2 b)$ & Risk Class & Primary treatment \\
\hline Leroy (France) & 2017 & $\mathrm{R}$ & 23 & $10.38(2.34-57)$ & $2 / 3$ & 7 & 82.5 & 4.3 & T2 & 65.2 & 30.4 & NR & EBRT, BT \\
\hline Fuller (USA) & 2020 & $P$ & 50 & NR & $N R$ & NR & $N R$ & $N R$ & NR & $N R$ & NR & NR & EBRT, BT, RP \\
\hline Jereczek-Fossa (Italy) & 2018 & $\mathrm{R}$ & 64 & $11.4(0.5-228.5)$ & $2 / 3$ & 7 & NR & NR & NR & NR & NR & NR & EBRT, BT \\
\hline Loi (Italy) & 2018 & $\mathrm{R}$ & 50 & $10(3.1-160)$ & NR & NR & 70 & 30 & NR & NR & NR & High & EBRT, RP+EBRT \\
\hline D'Agostino (Italy) & 2019 & $\mathrm{R}$ & 23 & $N R$ & NR & NR & NR & NR & NR & NR & NR & Intermediate & RP+EBRT, EBRT \\
\hline Pasquier (France) & 2019 & $\mathrm{R}$ & 100 & $10.2(2.3-120)$ & 1 & 6 & 93 & 7 & NR & NR & NR & Intermediate & EBRT \\
\hline Scher (France) & 2019 & $\mathrm{R}$ & 42 & $10.1(3-120)$ & $2 / 3$ & 7 & 82 & 18 & NR & NR & $N R$ & Intermediate & EBRT, RP+EBRT \\
\hline Cuccia (Italy) & 2020 & $\mathrm{R}$ & 24 & NR & 3 & 7 & 79 & 21 & NR & NR & NR & Intermediate & EBRT, BT \\
\hline Matrone (Italy) & 2020 & $\mathrm{R}$ & 44 & $8.7(2.6-46)$ & $2 / 3$ & $7 a$ & NR & NR & NR & $N R$ & $\mathrm{NR}$ & High & EBRT \\
\hline Caroli (Italy) & 2020 & $\mathrm{R}$ & 38 & NR & 2 & 7 & 100 & 0 & Т3 & 42.1 & 57.9 & NR & EBRT, RP+EBRT \\
\hline Bergamin (Australia) & 2020 & $P$ & 25 & $13(4.1-97)$ & 2 & 7 & 72 & 28 & T2a & 80 & 20 & Intermediate & EBRT, BT \\
\hline
\end{tabular}

$B T$, brachytherapy; HDR, high dose rate; LDR, low dose rate; $R$, retrospective; P, prospective; Pts, patients; $n$, number; PSA, prostate specific antigen; NR, not recorded; GS, Gleason score; EBRT, external beam radiotherapy; PBT, proton beam treatment; $R P$, radical prostatectomy.

For PSA, ISUP and GS, the median scores are presented.

TABLE 5 | Pre-salvage therapy disease and treatment characteristics for EBRT studies.

\begin{tabular}{|c|c|c|c|c|c|c|c|c|c|c|c|c|}
\hline First author/ country & Year & Design & TRS (mo)(range) & BCR definition & Age (years)(range) & PSA (ng/mL)(range) & ISUP & GS & $\%$ GS $(\leq 7)$ & $\%$ GS $(\geq 8)$ & Imaging for relapse & Biopsy \\
\hline Leroy (France) & 2017 & $\mathrm{R}$ & $65(28-150)$ & Phoenix & $70(58-82)$ & $2.5(0-11.7)$ & NR & NR & NR & NR & C-PET, MRI & Yes (83\%) \\
\hline Fuller (USA) & 2020 & $\mathrm{P}$ & $98(31-241)$ & Phoenix & $74(50-89)$ & $3.97(0.1-48.2)$ & 3 & 7 & 64 & 36 & MRI & Yes (100\%) \\
\hline Jereczek-Fossa (Italy) & 2018 & $\mathrm{R}$ & $99.7(23-208)$ & Phoenix & $73.2(52.6-81.7)$ & $3.89(0.17-51.8)$ & $2 / 3$ & 7 & NR & NR & C-PET, MRI, CT & Yes (44\%) \\
\hline Loi (Italy) & 2018 & $\mathrm{R}$ & $76(9-205)$ & Phoenix & $76(62-86)$ & $2.6(1-30)$ & $N R$ & NR & $N R$ & $N R$ & C-PET, MRI & $\mathrm{NR}$ \\
\hline D'Agostino (Italy) & 2019 & $\mathrm{R}$ & $90(26-138)$ & NR & $78(69-85)$ & $3.2(1.2-13.5)$ & NR & NR & NR & NR & C-PET & No \\
\hline Pasquier (France) & 2019 & $\mathrm{R}$ & $90(24-216)$ & Phoenix & $71.2(56-86)$ & $4.3(2.0-38.3)$ & 3 & 7 & 66 & 34 & C-PET, MRI & Yes (100\%) \\
\hline Scher (France) & 2019 & $\mathrm{R}$ & $82.5(29-207)$ & Phoenix & $64(49-77)$ & $3.1(0.01-23.7)$ & NR & NR & NR & NR & C-PET, MRI & Yes (80\%) \\
\hline Cuccia (Italy) & 2020 & $\mathrm{R}$ & $69(29-141)$ & Phoenix & $75(65-89)$ & $1.79(0.18-10)$ & NR & NR & NR & NR & C-PET/ PSMA-PET, MRI & No \\
\hline Matrone (Italy) & 2020 & $\mathrm{R}$ & $60(16.9-615.5)$ & Phoenix & $76(56-89)$ & $2.6(2-7.68)$ & 1 & 6 & NR & NR & MRI, C-PET & Yes (11\%) \\
\hline Caroli (Italy) & 2020 & $\mathrm{R}$ & NR & Phoenix & $75(71-80)$ & $1.1(0.82-2.59)$ & NR & NR & NR & NR & PSMA-PET & NR \\
\hline Bergamin (Australia) & 2020 & $\mathrm{P}$ & $99.6(54-163.2)$ & Phoenix & $72(62-83)$ & $4.1(1.1-16.6)$ & NR & NR & NR & NR & PSMA-PET & Yes (100\%) \\
\hline
\end{tabular}

$B T$, brachytherapy; HDR, high dose rate; LDR, low dose rate; PSA, prostate specific antigen; NR, not recorded; GS, Gleason score; TRS, median time from primary treatment to salvage therapy; mo, months; BCR, biochemical recurrence; ASTRO, American Society for Radiation Oncology; MRI, magnetic resonance imaging; NS, bone scan; CT, computed tomography; US, ultrasound; C-PET, Choline positron emission tomography; PSMA , prostate specific membrane antigen. For TRS, age, PSA, ISUP and GS, the median scores are presented. 
TABLE 6 | Salvage therapy details for BT studies.

\begin{tabular}{|c|c|c|c|c|c|c|c|c|c|c|c|c|}
\hline $\begin{array}{l}\text { First author } \\
\text { (country) }\end{array}$ & Year & $\begin{array}{l}\text { Single-centre (1) or } \\
\text { Multi-centre (2) }\end{array}$ & $\begin{array}{l}\text { Patients } \\
\text { (n) }\end{array}$ & BT Technique & $\begin{array}{l}\text { Radiation } \\
\text { Source }\end{array}$ & $\begin{array}{l}\text { Focal or } \\
\text { Whole- } \\
\text { gland }\end{array}$ & $\begin{array}{l}\text { Dose (total dose (Gy)/ dose per } \\
\text { fraction/ number of fractions) }\end{array}$ & $\begin{array}{l}\text { Duration of } \\
\text { treatment }\end{array}$ & $\begin{array}{l}\text { Adjuvant } \\
\text { ADT }\end{array}$ & $\begin{array}{l}\text { Follow-up } \\
\text { (mo) (range) }\end{array}$ & BC (\%) & Oncologic outcomes \\
\hline B Lee (USA) & 2007 & 1 & 21 & HDR & $\mid r-192$ & Whole & $36 / 6 / 6$ & 7 days & No & 18.7 & 90.8 & 2-yr bRFS 89\% \\
\hline Lyczek (Poland) & 2009 & 1 & 115 & HDR & $\mid r-192$ & Whole & $30 / 10 / 3$ & 9 weeks & NR & NR & $\begin{array}{c}46 \text { (PSA<6) } \\
\text { vs } 18 \\
(\text { PSA }>6)\end{array}$ & $\begin{array}{l}\text { OS } 86 \%(P S A<6) \text { vs } 48 \% \\
(\text { PSA>6) }\end{array}$ \\
\hline Chen (USA) & 2013 & 1 & 52 & HDR & $\mid r-192$ & Whole & $36 / 6 / 6$ & 10 days & NR & $\begin{array}{c}59.6(5.9- \\
154.7)\end{array}$ & 55.7 & 5-yr bRFS 51\%, 5-yr OS 92\% \\
\hline Kukielka (Poland) & 2014 & 1 & 25 & $\begin{array}{l}\text { HDR with } \\
\text { interstitial } \\
\text { hyperthermia }\end{array}$ & $\mid r-192$ & Whole & 37924 & 63 days & Yes (12\%) & $13(4-48)$ & NR & 2-yr bRFS 74\% \\
\hline *Yamada (USA) & 2014 & 1 & 42 & HDR & $\mid r-192$ & Whole & $32 / 8 / 4$ & 30 hours & Yes (43\%) & $36(2-66)$ & 68.5 & 5 -yr OS 90.3\% \\
\hline Jiang (Germany) & 2016 & 1 & 29 & HDR & $\mid r-192$ & Whole & $30 / 10 / 3$ & 3 weeks & $\begin{array}{c}\text { Yes } \\
(34.5 \%)\end{array}$ & $73(61-140)$ & 45 & 5-yr bRFS 45\%, 5-yr OS 95.5\% \\
\hline Lacy (USA) & 2016 & 1 & 21 & HDR & $\mid r-192$ & Whole & 108-144 Gy & - & $\begin{array}{c}\text { Yes } \\
(14.3 \%)\end{array}$ & $61(10-149)$ & 47.6 & NR \\
\hline Wojcieszek & 2016 & 1 & 83 & HDR & $\mid r-192$ & Whole & $30 / 10 / 3$ & 28-30 days & Yes $(53 \%)$ & $41(11-76)$ & 67 & 5 -yr CSS 87\% \\
\hline Lopez (Spain) & 2019 & 2 & $\begin{array}{l}75 \\
44\end{array}$ & $\begin{array}{l}\text { HDR } \\
\text { LDR }\end{array}$ & $\begin{array}{l}\text { Ir-192 } \\
\text { NR }\end{array}$ & $\begin{array}{l}\text { Whole } \\
\text { Whole }\end{array}$ & $\begin{array}{l}32 / 7-10 / 2-4 \\
145 \text { Gy }\end{array}$ & $\begin{array}{l}- \\
-\end{array}$ & $\begin{array}{l}\text { Yes }(45 \%) \\
\text { Yes } \\
(532 \%)\end{array}$ & $\begin{array}{l}52 \\
52\end{array}$ & $\begin{array}{l}67.5 \\
68\end{array}$ & $\begin{array}{l}5 \text {-yr bRFS 65\% } \\
5 \text {-yr bRFS 79\% }\end{array}$ \\
\hline $\begin{array}{l}\text { Chitmanee (UK) } \\
\text { Slevin (UK) }\end{array}$ & 2020 & 1 & 50 & HDR & $\mid r-192$ & Focal & 1 x 19 Gy & - & Yes $(8 \%)$ & $21(1-53)$ & 46 & 2-yr bRFS 63\%, 3-yr bRFS 46\% \\
\hline $\begin{array}{l}\text { Sevin (UK) } \\
\text { van Son } \\
\text { (Netherlands) }\end{array}$ & 2020 & 1 & $\begin{array}{l}43 \\
50\end{array}$ & $\begin{array}{l}\text { HDR (MRI } \\
\text { Guided ultra- }\end{array}$ & $\begin{array}{l}\mid l r-192 \\
\mid r-192\end{array}$ & Ultra-focal & $\begin{array}{l}1 \times 19 \text { Gy } \\
1 \times 19 \text { Gy }\end{array}$ & - & $\begin{array}{l}\text { Yes }(14 \%) \\
\text { Yes }(12 \%)\end{array}$ & $\begin{array}{l}26(1-60) \\
31(13-58)\end{array}$ & 48 & $\begin{array}{l}2.5 \text { yr bRFS } 51 \% \text {, mFS } 75 \% \text {, OS } \\
98 \%\end{array}$ \\
\hline \multirow[t]{2}{*}{ Kollmeier (USA) } & 2017 & 1 & 37 & LDR & $\begin{array}{l}125-1(8 \%) \text { or } \\
103-P d(92 \%)\end{array}$ & Whole & 125-144 Gy & - & Yes (46\%) & $31(2-97)$ & 65 & $\begin{array}{l}\text { 3-yr bRFS 60.2\%. 3-yr mFS } \\
78.7 \%\end{array}$ \\
\hline & & & 61 & HDR & |r-192 & Whole & $\begin{array}{l}32 / 8 / 4(n=58), 28 / 7 / 4 \text { / }(n=1) \\
\quad \text { and } 22 / 11 / 2 \text { (n=1) }\end{array}$ & 30 hours & Yes (44\%) & & & \\
\hline Baumann (USA) & 2017 & 1 & 33 & HDR/LDR & $\begin{array}{l}\text { 103-Pd (LDR) } \\
\text { and Ir-192 } \\
\text { (HDR) }\end{array}$ & Whole & LDR (90-100 Gy) or HDR (30/6/5) & NR & $\begin{array}{l}\text { Yes } \\
(100 \%)\end{array}$ & $61(7-150)$ & 67 & 7-yr RFS 67\% \\
\hline Henriquez (Spain) & 2014 & 1 & 56 & HDR/LDR & $|r-192 / 125-|$ & Whole & HDR: 50.5 / 5.25 / 1-4, LDR: 145 Gy & NR & $\begin{array}{c}\text { Yes } \\
(26.8 \%)\end{array}$ & $48(25-109)$ & NR & 5-yr bRFS 77\%, 5-yr OS 70\% \\
\hline Grado (USA) & 1999 & 1 & 49 & LDR & $\begin{array}{l}125-\mathrm{I}(76 \%) \text { or } \\
103-\operatorname{Pd}(24 \%)\end{array}$ & Whole & 80-180 Gy & - & Yes $(16 \%)$ & $\begin{array}{l}41.7(21.8- \\
185.2)\end{array}$ & 34 & \multirow{8}{*}{$\begin{array}{l}\text { 3-yr bRFS 48\%, 5-yr bRFS 34\%. } \\
\text { LC 98\% } \\
\text { 3-yr bRFS 83.9\%, } \\
\text { 5-yr bRFS 41.9\% } \\
4 \text {-yr bRFS 70\% } \\
\text { 5-yr bRFS 38\%, 5-yr OS 81\% } \\
\text { 3-yr bRFS 89.5\% 3-year CSS } \\
\text { 96\% } \\
\text { 5-yr bRFS 65\%, 5-yr CSS 94\%, } \\
5 \text {-yr OS 96\% } \\
\text { 1-yr bRFS 51\%, 5-yr bRFS 20\%, } \\
\text { 5-yr CSS 74\%, 5-yr OS 72\% } \\
\text { 3-yr bRFS 71\% }\end{array}$} \\
\hline Koutrouvelis (USA) & 2003 & 1 & 31 & LDR & $\begin{array}{l}125-\mathrm{I}(77 \%) \text { or } \\
103-\operatorname{Pd}(23 \%)\end{array}$ & Whole & 100-144 Gy & - & No & $30(12-84)$ & 87 & \\
\hline Nguyen (USA) & 2007 & 1 & 25 & LDR & $125-1$ & Whole & 137 Gy & - & No & $47(14-75)$ & 72 & \\
\hline HK Lee (USA) & 2008 & 1 & 21 & LDR & 103-Pd & Whole & 90 Gy & - & Yes (57\%) & 36 & NA & \\
\hline Aaronson (USA) & 2009 & 1 & 24 & LDR & $125-$ I or 103-Pd & Whole & 146 Gy & - & Yes (29\%) & $30(13-65)$ & 87.5 & \\
\hline Burri (USA) & 2010 & 1 & 37 & LDR & \multirow{8}{*}{ 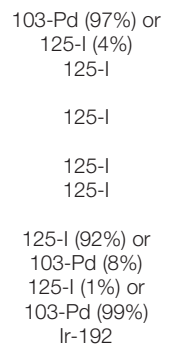 } & Whole & 110-135 Gy & - & Yes (84\%) & $86(2-156)$ & NA & \\
\hline $\begin{array}{l}\text { Moman } \\
\text { (Netherlands) }\end{array}$ & 2010 & 1 & 31 & LDR & & Whole & 145 Gy & - & NA & 108 & 19 & \\
\hline $\begin{array}{l}\text { Peters } \\
\text { (Netherlands) }\end{array}$ & 2014 & 1 & 20 & LDR & & Focal & 144 Gy & - & NR & $36(10-45)$ & 71 & \\
\hline Vargas (USA) & 2014 & 1 & 69 & LDR & & Whole & 100 Gy & - & Yes $(90 \%)$ & $60(7-164)$ & 68.6 & \multirow{3}{*}{$\begin{array}{l}\text { 5-yr OS 64\%, 5-yr mFS 90\% } \\
\text { Estimated 10-yr PCaSS 43\%, } \\
10 \text {-yr OS 34\% } \\
\text { NR }\end{array}$} \\
\hline $\begin{array}{l}\text { Peters } \\
\text { (Netherlands) }\end{array}$ & 2016 & 2 & 62 & $\begin{array}{l}\text { LDR (Whole } \\
\text { Gland) }\end{array}$ & & Whole & 145 Gy & - & Yes (34\%) & $78(5-139)$ & NR & \\
\hline Crook (Canada) & 2019 & 2 & 92 & LDR & & Whole & $120-140$ Gy & - & NR & 54 & NR & \\
\hline Smith (USA) & 2020 & 2 & 108 & LDR & & Whole & 100 Gy & - & $\begin{array}{c}\text { Yes } \\
(93.5 \%)\end{array}$ & $75(1-228)$ & NR & \multirow{2}{*}{$\begin{array}{l}\text { 5-yr bRFS 63\%, } 10-y \text {-y bRFS } \\
52 \% \\
5-\text { yr bRFS } 65.6 \%, \text { LC 86.6\% }\end{array}$} \\
\hline Schonle (Germany) & 2020 & 1 & 82 & PDR & & Whole & $60 / 30$ / 2 & 4 weeks & Yes (43.9) & $49(12-129)$ & 65.6 & \\
\hline
\end{tabular}

BT, brachytherapy; HDR, high dose rate; LDR, low dose rate; 125-I, Iodine-125; 103-Pd, Palladium-103; Ir-192, Iridium-192; Gy, Gray; ADT, androgen deprivation therapy; mo, months; BC, biochemical control; bRFS, biochemical recurrence free survival; mFS, metastasis free survival; RFS, relapse free survival; CSS, cancer specific survival; OS, overall survival.

Yamada (USA) study cohort included in further publication Kollmeier (USA) however specific treatment characteristics and toxicity were not covered in later paper. 


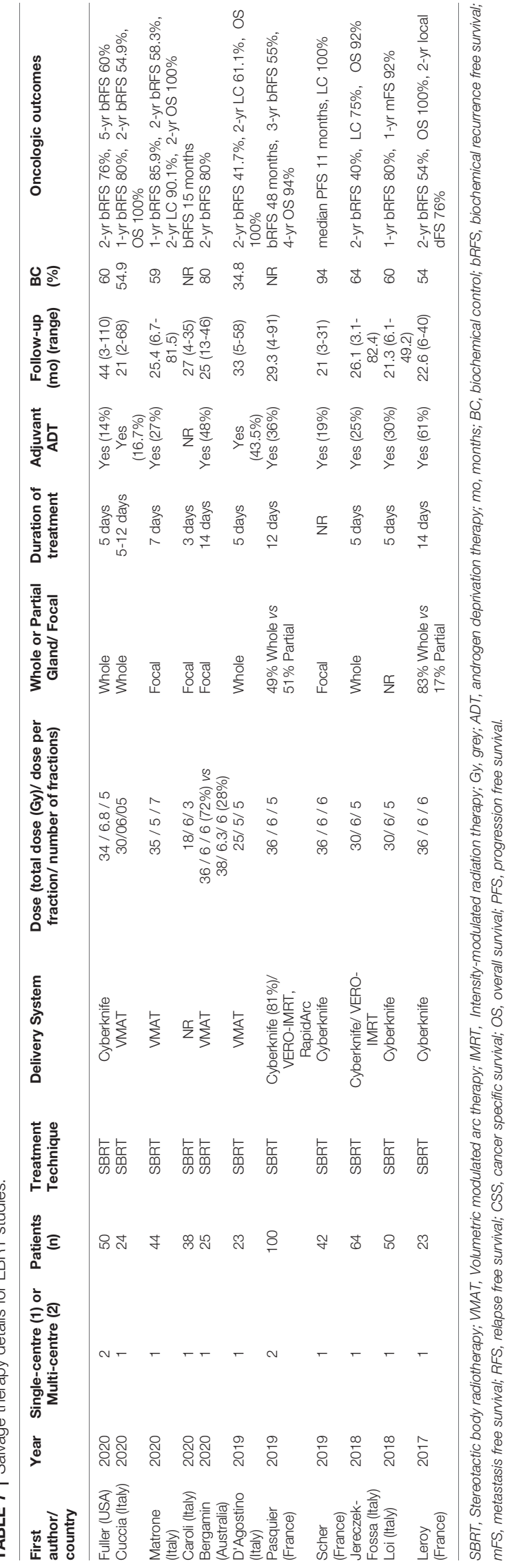

28 BT studies, 24 included only patients with biopsy-proven local recurrence. Three of 11 SBRT studies included patients with histological confirmation of recurrence.

For BT studies, median follow up duration (range) was 47.5 months (13-108) compared with 25.4 months (21-44) for SBRT studies. The use of ADT with salvage therapy ranged from 8$100 \%$ in the BT study group and $14-61 \%$ in the SBRT group.

\section{Oncological Outcomes}

For the LDR-BT studies, the median (range) 2-year and 5-year bRFS rates were $71 \%(48-89.5 \%)$ and $52.5 \%(20-79 \%)$. For the HDR-BT studies, the median (range) 2-year and 5-year bRFS rates were $74 \%(63-89 \%)$ and $51 \%(45-65 \%)$. For the SBRT studies, the median (range) 2-year bRFS for the SBRT group was $54.9 \%(40-80 \%)$. A 5 -year estimate of bRFS following SBRT was only available for one study and was $60 \%$ (47). For focal gland BT, the median (range) 3-year bRFS was 63\% (42-71\%). For focal SBRT, the median (range) 3-year bRFS was 69\% (58-80\%). 3year bRFS was presented as 2-year bRFS was not reported by the majority of these focal RT studies.

\section{TOXICITY}

A summary of clinician reported acute and late GU and GI toxicity data for each study is presented in Table 8 (BT) and Table 9 (SBRT).

In studies that only included LDR-BT, mean (range) grade 3 or higher toxicities were 7.4\% (0-14\%) (acute GU), 13.6\% (030\%) (late GU), 6.5\% (0-19\%) (acute GI) and 6.4\% (0-20\%) (late GI). In studies that only included HDR-BT, mean (range) grade 3 or higher toxicities were $2 \%(0-14 \%)$ (acute GU), $7.9 \%(0$ $21.3 \%$ ) (late GU) and $0.1 \%(0-0.9 \%)$ (late GI). No grade 3 or higher acute GI toxicity was reported. For the SBRT group, mean (range) grade 3 or higher toxicities were 1.8\% (0-8.7\%) (acute GU), $2.7 \%(0-8 \%)$ (late GU), $0.5 \%(0-4 \%)$ (acute GI) and $0.2 \%$ (0-1.5\%) (late GI).

For the focal gland BT group $(n=4)(16,34,36,38)$, the only grade 3 or higher toxicity reported was late GU toxicity - with a mean (range) of $4.8 \%(2-10 \%)$. For the focal gland SBRT group $(\mathrm{n}=4)(46,51,53,54)$, mean (range) grade 3 or higher toxicities were $0.5 \%(0-2 \%)$ (acute GU), $1.5 \%$ (0-4\%) (late GU) and $1 \%(0$ $4 \%$ ) (acute GI). No late GI toxicity was reported.

Symptoms of erectile dysfunction were specifically reported by nine BT studies (32\%) and one SBRT study (9\%).

Thirteen studies (33.3\%) (12 BT and one SBRT study) included PROMs, with the most common assessment tool used being the international prostate symptom score (IPSS).

\section{DISCUSSION}

This systematic review evaluated the most up-to-date evidence for salvage BT and SBRT and found that both treatment options provide good biochemical control with acceptable late GU/GI toxicity. However there is considerable heterogeneity between 
TABLE 8 | Toxicity details for BT studies.

\begin{tabular}{|c|c|c|c|c|c|c|c|c|c|c|c|}
\hline \multirow[t]{2}{*}{ First author (country) } & \multirow[t]{2}{*}{ Toxicity Scale } & \multicolumn{2}{|c|}{ Acute GU toxicity } & \multicolumn{2}{|c|}{ Acute GI toxicity } & \multicolumn{2}{|c|}{ Late GU toxicity } & \multicolumn{2}{|c|}{ Late GI toxicity } & \multirow[t]{2}{*}{ Erectile Dysfunction } & \multirow[t]{2}{*}{ PROMS } \\
\hline & & Grade $\leq 2$ & Grade $\geq 3$ & Grade $\leq 2$ & Grade $\geq 3$ & Grade $\leq 2$ & Grade $\geq 3$ & Grade $\leq \mathbf{2}$ & Grade $\geq 3$ & & \\
\hline Kollmeier (USA) & CTCAE v4.0 & $96.0 \%$ & - & $96.0 \%$ & - & $82.0 \%$ & $9.0 \%$ & $91.0 \%$ & $2.0 \%$ & NR & Yes (IPSS) \\
\hline Baumann (USA) & CTCAE v4.0 & $82.0 \%$ & - & $9.0 \%$ & - & $42.0 \%$ & $12.0 \%$ & $3.0 \%$ & - & NR & Yes (IPSS) \\
\hline Wojcieszek (Poland) & CTCAE v4.0 & $87.0 \%$ & $1.0 \%$ & $6.0 \%$ & - & $72.0 \%$ & $13.0 \%$ & $6.0 \%$ & - & $N R$ & NR \\
\hline *Yamada (USA) & CTCAE v3.0 & $78.0 \%$ & - & NR & $N R$ & $86.0 \%$ & $10.0 \%$ & $57.0 \%$ & - & Yes & Yes (IPSS) \\
\hline Peters (Netherlands) & CTCAE v4.0 & $100.0 \%$ & - & $55.0 \%$ & - & $40.0 \%$ & $5.0 \%$ & $35.0 \%$ & - & Yes (80\%) & Yes (RAND-36, EORTC) \\
\hline Vargas (USA) & NR & $5.0 \%$ & $8.7 \%$ & NR & NR & $5.0 \%$ & $8.7 \%$ & $7.0 \%$ & $3.0 \%$ & $N R$ & NR \\
\hline Chen (USA) & CTCAE v4.0 & $98.0 \%$ & $2.0 \%$ & $100.0 \%$ & - & $98.0 \%$ & $2.0 \%$ & $100.0 \%$ & - & Yes (81\%) & $N R$ \\
\hline Burri (USA) & CTCAE v3.0 & $35.0 \%$ & $11.0 \%$ & $5.0 \%$ & NR & $35.0 \%$ & $11.0 \%$ & NR & $3.0 \%$ & Yes (75\%) & NR \\
\hline Moman (Netherlands) & CTCAE v3.0 & $87.0 \%$ & $3.0 \%$ & $55.0 \%$ & - & $55.0 \%$ & $19.0 \%$ & $51.0 \%$ & $6.0 \%$ & $\mathrm{NR}$ & NR \\
\hline Aaronson (USA) & CTCAE V3.0 & NR & NR & $N R$ & $3.0 \%$ & $37.0 \%$ & $4.0 \%$ & NR & $4.0 \%$ & $\mathrm{NR}$ & Yes (IPSS, IIEF-5) \\
\hline HK Lee (USA) & RTOG & $29.0 \%$ & - & $5.0 \%$ & - & $29.0 \%$ & - & $5.0 \%$ & - & $N R$ & NR \\
\hline Nguyen (USA) & RTOG & NR & NR & NR & NR & NR & $20.0 \%$ & NR & $20.0 \%$ & NR & NR \\
\hline B Lee (USA) & CTCAE v3.0 & $86.0 \%$ & $14.0 \%$ & $14.0 \%$ & - & NR & $5.0 \%$ & - & - & Yes (95\%) & NR \\
\hline Koutrouvelis (USA) & NR & $13.0 \%$ & $13.0 \%$ & $13.0 \%$ & $19.0 \%$ & $13.0 \%$ & $13.0 \%$ & $13.0 \%$ & $19.0 \%$ & NR & $N R$ \\
\hline Grado (USA) & NR & NR & NR & NR & NR & $10.0 \%$ & $20.0 \%$ & $4.0 \%$ & $2.0 \%$ & NR & NR \\
\hline Slevin (UK) & CTCAE v4.0 & $91.0 \%$ & - & $14.0 \%$ & - & $65.0 \%$ & $2.0 \%$ & $14.0 \%$ & - & NR & NR \\
\hline \multirow[t]{2}{*}{ Lopez (Spain) } & RTOG & $33.0 \%$ & $N R$ & NR & $N R$ & NR & $21.3 \%$ & NR & $N R$ & $N R$ & $N R$ \\
\hline & RTOG & $33.0 \%$ & NR & NR & NR & $N R$ & $27.3 \%$ & $N R$ & $N R$ & $N R$ & NR \\
\hline Crook (Canada) & CTCAE v3.0 & NR & $14.0 \%$ & NR & $14.0 \%$ & $N R$ & $7.0 \%$ & $N R$ & $4.0 \%$ & $\mathrm{NR}$ & Yes (IPSS) \\
\hline Smith (USA) & CTCAE v5.0 & NR & NR & NR & NR & NR & $15.7 \%$ & NR & $2.8 \%$ & Yes (80\%) & Yes (IPSS, MSEFS) \\
\hline Kukielka (Poland) & CTCAE v4.0 & $96.0 \%$ & - & $12.0 \%$ & - & $41.0 \%$ & - & - & - & $\mathrm{NR}$ & Yes (IPSS) \\
\hline Schonle (Germany) & CTCAE v4.0 & $15.8 \%$ & $6.1 \%$ & $2.4 \%$ & - & $15.8 \%$ & $6.1 \%$ & $2.4 \%$ & - & NR & $\mathrm{NR}$ \\
\hline Chitmanee (UK) & NR & $90.0 \%$ & - & $32.0 \%$ & - & $72.0 \%$ & $10.0 \%$ & $30.0 \%$ & - & Yes (86\%) & Yes (IPSS) \\
\hline Henriquez (Spain) & CTCAE v3.0 & NR & NR & NR & NR & NR & $23.0 \%$ & NR & $4.0 \%$ & NR & NR \\
\hline Peters (Netherlands) & CTCAE v4.03 & NR & $N R$ & NR & $N R$ & NR & $30.0 \%$ & NR & $10.0 \%$ & NR & NR \\
\hline Jiang (Germany) & CTCAE v4.0 & $100.0 \%$ & - & $100.0 \%$ & - & $90.9 \%$ & $9.0 \%$ & $100.0 \%$ & - & NR & Yes (IPSS) \\
\hline van Son (Netherlands) & CTCAE v4.0 & $65.0 \%$ & - & $37.0 \%$ & - & $55.0 \%$ & $2.0 \%$ & $37.0 \%$ & - & Yes (100\%) & Yes (IPSS, RAND-36) \\
\hline Lacy (USA) & RTOG & $9.6 \%$ & $9.6 \%$ & $9.6 \%$ & $9.6 \%$ & $9.6 \%$ & $9.6 \%$ & $9.6 \%$ & $9.6 \%$ & Yes (45.5\%) & Yes (IPSS) \\
\hline Lyczek (Poland) & RTOG & $29.6 \%$ & $2.6 \%$ & $7.9 \%$ & $N R$ & $7.0 \%$ & $12.2 \%$ & $1.7 \%$ & $0.9 \%$ & NR & NR \\
\hline
\end{tabular}

- , O\% reported toxicity; NR, not reported; GU, genitourinary; Gl, gastrointestinal; CTCAE, Common Terminology Criteria for Adverse Events; RTOG, Radiation Therapy Oncology Group; NR, not reported; PROMS, patient recorded outcome measures; IPSS, International prostate symptom score; RAND-36, RAND-36 Health Survey; EORTC, European Organisation for Research and Treatment of Cancer Quality of Life questionnaire; IIEF-5, International Index of Erectile Function questionnaire; MSEFS, Mount Sinai Erectile Function Score.

Yamada (USA) study cohort included in further publication Kollmeier (USA) however specific treatment characteristics and toxicity were not covered in later paper. 


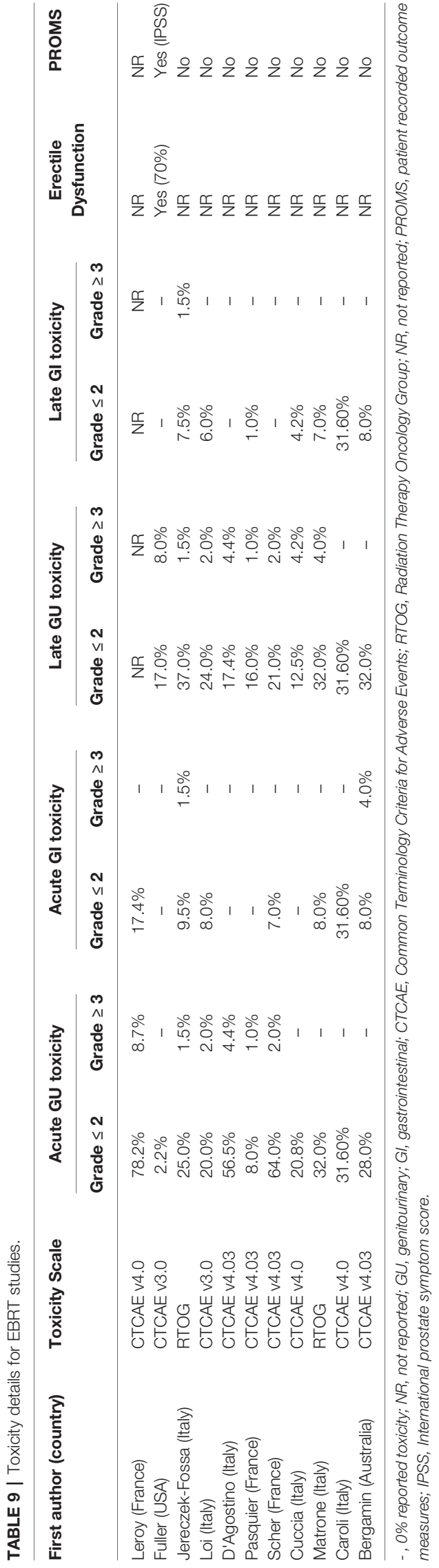

studies for numbers of patients, risk groups of included patients, evaluated treatments, reported endpoints, duration of follow up and methods of toxicity assessment (clinician-assessed versus PROMs). The quality of studies was low and meta-analysis was therefore not conducted due to the significant bias associated with these uncontrolled studies. This highlights the need for further high quality prospective and randomised studies to measure the efficacy and toxicity associated with salvage irradiation.

Consensus national and international recommendations for reirradiation are limited. The European Association of Urology (EAU) guidelines recommend salvage reirradiation using $\mathrm{BT}$ or SBRT for locally recurrent prostate cancer should only be undertaken in a clinical trial setting (55). American Society for Radiation Oncology (ASTRO) and National Comprehensive Cancer Network (NCCN) clinical practice guidelines does not comment on the use of reirradiation for prostate cancer however both the European Society of Radiation Oncology (ESTRO) and American Society of Brachytherapy (ABS) recommendations on prostate HDR-BT highlight the accumulating evidence for salvage HDR-BT in local recurrence as showing great promise (56-58).

There has been increasing interest in the use of salvage therapies for locally recurrent prostate cancer after primary radiation, although concerns have been raised regarding the potential for severe late toxicity (59). Both BT and SBRT, show durable outcomes in terms of biochemical control with reasonable reported toxicities in the majority of reviewed studies. However, inconsistencies in reporting and missing data preclude accurate comparison between these studies, which are mainly composed of case series. Longer term efficacy data and duration of follow up was available for more BT studies than SBRT but, at short term follow-up, the clinician-reported toxicity following salvage SBRT appear to be infrequent (60).

Two previous meta-analyses which compared salvage therapies in recurrent prostate cancer have been conducted, which included radical prostatectomy, cryotherapy and HIFU in addition to BT and EBRT $(10,61)$. The meta-analysis by Valle et al. (61) reported that recurrence free survival and toxicity rates were best for salvage radiotherapeutic modalities compared to other salvage treatments, and BT appeared to offer the best balance between toxicity and efficacy. For example, the estimated recurrence free survival at 2 years for BT was $77-79 \%$ compared to $52-72 \%$ for cryotherapy, HIFU and salvage radical prostatectomy. In addition, lower grade 3 or higher GU toxicity was observed $(5-10 \%$ versus $20 \%$ for BT compared with other salvage therapies) (61). The quality of the evidence was not assessed and sub-group and sensitivity analyses to explore potential impact of clinical heterogeneity were also not specified in this meta-analysis (61). In addition, it was unclear how many studies were excluded from the meta-analysis due to incompatible definitions, outcome measures and follow-up periods. Interestingly, the 2-year bRFS of SBRT (54.9\%) appeared to be lower than both LDR- and HDR-BT (71\% and $74 \%$ respectively). A formal comparison between these modalities is limited by confounding factors, although these data raise an interesting question as to whether salvage SBRT could be inferior to BT in terms of biochemical control. 
Comparing the SBRT studies to BT remains challenging in view of the heterogeneous populations and shorter follow-up available for SBRT with only one study providing 5-yr bRFS data (although in this study, comparable to outcomes from salvage BT were reported) (47). No prospective randomised studies exist which compare BT and SBRT as salvage therapies for locally recurrent prostate cancer and this is ultimately what is required.

There may be dosimetric advantages with the use of BT compared with SBRT. A previous planning study in the primary disease setting concluded that HDR-BT was able to achieve higher intraprostatic doses and greater sparing of the rectum than SBRT (62). It is possible that developments in SBRT planning and delivery might lead to improved outcomes. For example, the superior soft tissue visualisation and functional imaging capabilities of MR guided SBRT might permit better delineation of tumour, greater accuracy of treatment delivery and offer opportunities for dose escalation (63). Whether this would translate into a clinical benefit at this point remains uncertain. There remains considerable interest in salvage SBRT as evidenced by the fact that 8 of 11 SBRT studies were published in the last two years.

Based on the studies evaluated in this review, salvage LDR-BT appeared to have the potential for higher grade $3+$ toxicity compared to HDR-BT $(19,24)$. In a study which used PROMs, LDR-BT had a higher peak change in IPSS in the early postimplant period and a higher peak urinary symptom flare at 12 months compared with HDR-BT, although the majority of these scores returned to baseline 2-3 years post-treatment (40). There have been no prospective studies comparing these techniques in the reirradiation setting. In the primary treatment setting however prospective and randomised studies have shown HDR-BT to have better quality of life scores compared to LDR-BT in the acute post-treatment phase, particularly in the urinary health domain, which suggests that HDR was better tolerated $(64,65)$. Similarly, evidence from registries and randomised trials of LDR/EBRT combination therapy and HDR/EBRT combination therapy in the primary disease setting suggest that LDR/EBRT might be associated with higher incidence of significant late GU toxicity although no direct comparison has been performed between the two treatments (66-68).

Based on the available data, grade 3 or higher GU and GI toxicity with SBRT was rare, although follow-up beyond 2 years is limited $(9,45-54)$. SBRT has the potential to limit the risk of severe late GU/GI toxicity compared with less conformal EBRT techniques (69). Careful patient selection remains vital, especially for those at greater risk of excess toxicity following salvage therapy. In a recent observational series of salvage SBRT, grade $3+\mathrm{GU}$ toxicity was disproportionately observed in patients treated with $\mathrm{BT}$ or radical prostatectomy plus salvage RT in the primary disease setting (47). Furthermore, the use of focal salvage techniques with BT and SBRT appear to have lower toxicity rates and comparable bRFS rates however this is limited to a number of uncontrolled, single-arm case series $(16,34,36$, $38,46,51,53,54)$.

Appropriate patient selection for salvage RT treatments is vital. The European Society for Radiotherapy and Oncology
Advisory Committee on Radiation Oncology Practice (ESTRO ACROP) recently conducted a Delphi consensus of expert opinion on patient selection criteria for salvage RT (70). Selection criteria with high levels of agreement $(>80 \%)$ included Eastern Cooperative Oncology Group performance status of $0-1$, satisfactory urinary flow with a known IPSS prior to salvage and use of PET-CT to exclude metastatic disease and MRI to define the target. Agreement was reached that concomitant ADT with salvage RT was unnecessary and that previous ADT use was not a contraindication to salvage RT. It was also recommended that the primary RT dose should be taken into account when considering salvage SBRT. In terms of time duration between primary RT and salvage RT, although consensus was not achieved a minimum interval of 2 years reached major agreement (defined as $65-80 \%$ agreement).

The impact on quality of life has not been well assessed in the salvage radiotherapy setting with only a third of studies in this systematic review including PROMs. Only one of 11 SBRT (9.1\%) studies included PROMs. Without this information, it is likely that reported rates of toxicity are underestimated (71). Assessment of residual toxicity following primary treatment using validated PROM instruments such as Expanded Prostate Cancer Index (EPIC) could be an important tool for identification of patients at risk of significant toxicity from salvage therapies. Integration of longitudinal PROM assessment into clinical trials is important to ascertain the time-dependent nature of toxicity onset/resolution after treatment $(71,72)$.

The role of ADT with salvage BT/EBRT remains unclear and no consensus could be reached during a previous Delphi consensus (73). The use of ADT with salvage radiation therapy in the evaluated studies was highly variable (8-100\%) and reporting of ADT duration was incomplete $(16,32,36,41,74)$. Several BT studies did not report ADT usage or did not use neoadjuvant ADT $(24,25,33)$. Salvage therapies may delay the need for ADT, with up to $69 \%$ patients remaining free of ADT at 5 years following salvage SBRT (47). Some authors view salvage $\mathrm{BT} / \mathrm{SBRT}$ as ADT sparing, which might have the potential to improve quality of life (75).

A recent study found that only $15 \%$ of relapses following salvage BT were solely in the prostate (36), suggesting most are likely to be systemic failure therefore accurate and consistent whole body imaging staging is imperative. The optimal combination of re-staging imaging following biochemical failure after primary treatment, and the most clinically relevant PSA level at which to trigger such imaging, remains uncertain (76). Despite the poor accuracy of CT and isotope bone scintigraphy, $21 \%$ of studies in this systematic review used these modalities for restaging and patient selection. It is possible that some patients in these studies could have had undiagnosed metastatic disease, and this could be responsible for some subsequent biochemical failures $(75,77)$. Less than half of studies used mpMRI and PET-CT for re-staging prior to salvage therapy. mpMRI has the potential to be particularly useful for detecting local recurrence following previous prostate radiotherapy, although studies evaluating its accuracy are limited (78). The use of novel imaging modalities such as 
Gallium-68 [ $\left.{ }^{68} \mathrm{Ga}\right]$ or Fluorine-18 $\left[{ }^{18} \mathrm{~F}\right]$ labelled PSMA PET-CT, may allow detection of local recurrence at lower PSA levels. While this could lead to a change in management for patients identified with recurrent disease, it was only used in $10 \%$ of the studies in this systematic review (79). ${ }^{68}$ Ga-PSMA PET-CT has been shown to demonstrate recurrences at prostate-specific antigen (PSA) levels below the Phoenix definition of biochemical failure and it allows for both local staging and exclusion of distant metastatic disease in patients with biochemical failure (80). The recent proPSMA randomised study reported that PSMA PET-CT had a greater accuracy compared to conventional imaging with CT and bone scan in the primary setting (92\% vs 65\%) (81). PSMA PET-CT also has superior performance characteristics for the detection of distant metastasis in the setting of biochemical failure compared to other PET tracers (82). Nevertheless, the clinical significance of detecting and treating small volume local recurrence at low PSA levels remains uncertain and may risk additional toxicity. Prospective randomised trials comparing BT and SBRT for salvage treatment of locally recurrent prostate cancer are required to determine the efficacy/toxicity of these interventions

\section{SUMMARY OF MAIN FINDINGS}

- For the LDR-BT studies, the median (range) 2-year and 5year bRFS rates were 71\% (48-89.5\%) and 52.5\% (20-79\%).

- For the HDR-BT studies, the median (range) 2-year and 5year bRFS rates were 74\% (63-89\%) and 51\% (45-65\%).

- For the SBRT studies, the median (range) 2-year bRFS for the SBRT group was $54.9 \%$ (40-80\%).

- For LDR-BT, mean (range) grade 3 or higher toxicities were 7.4\% (0-14\%) (acute GU), 13.6\% (0-30\%) (late GU), 6.5\% (0$19 \%)$ (acute GI) and 6.4\% (0-20\%) (late GI).

- For HDR-BT, mean (range) grade 3 or higher toxicities were $2 \%(0-14 \%)$ (acute GU), 7.9\% (0-21.3\%) (late GU) and $0.1 \%$ $(0-0.9 \%)$ (late GI). No grade 3 or higher acute GI toxicity was reported.

- For SBRT, mean (range) grade 3 or higher toxicities were $1.8 \%(0-8.7 \%)$ (acute GU), 2.7\% (0-8\%) (late GU), 0.5\% (0$4 \%$ ) (acute GI) and $0.2 \%(0-1.5 \%)$ (late GI).

- Only thirteen studies (33.3\%) included PROMs, with the most common assessment tool used being IPSS.

\section{LIMITATIONS}

The overall quality of evaluated evidence was low. A metaanalysis was not conducted to quantitatively compare the studies as the majority of these were non-comparative retrospective case series with differences in baseline patient demographics, primary and/or salvage treatments, reported endpoints reported and use of ADT. This limits the conclusions that can be drawn about the effectiveness/toxicity of salvage BT/SBRT. High-quality data from prospective trials are still needed to validate the toxicity and long-term clinical outcomes associated with the salvage treatment of recurrent prostate cancer using BT or EBRT, following previous RT.

\section{CONCLUSIONS}

Salvage reirradiation of radiorecurrent prostate cancer using HDR-BT or SBRT provides similar biochemical control and acceptable late toxicity. Salvage LDR-BT is associated with higher late GU/GI toxicity. Challenges exist in comparing BT and SBRT from the current literature due to inconsistencies in reporting and missing data. Prospective randomised trials comparing BT and SBRT and assessing PROMs as well as cancer control outcomes in this setting are needed.

\section{DATA AVAILABILITY STATEMENT}

The original contributions presented in the study are included in the article/Supplementary Material. Further inquiries can be directed to the corresponding author.

\section{AUTHOR CONTRIBUTIONS}

All authors contributed to the article and approved the submitted version. JZ and FS did the literature search, assembly of data and data analysis. JZ, FS and AH: data interpretation to ensure relevance of findings.

\section{FUNDING}

JZ is a Clinical Research Fellow supported by a Cancer Research UK Award (Leeds-Manchester Stella Erdheim Clinical PhD Fellowship - Grant Reference Number 95653117/95653118). FS is a Clinical Research Fellow supported by a Cancer Research UK Centres Network Accelerator Award to the ARTNET Consortium (Grant number A21993). AS receives salary support for academic work from Cancer Research UK via the Radiation Research Centre of Excellence at the University of Leeds (Grant Reference Number C19942/A2882). AC and PH are supported by NIHR Manchester Biomedical Research Centre. AH is supported by grants from Cancer Research UK (award number 108036), National Institute for Health Research (NIHR) (award number 111218), Medical Research Council (MRC) (award number 107154) and Sir John Fisher Foundation.

\section{SUPPLEMENTARY MATERIAL}

The Supplementary Material for this article can be found online at: https://www.frontiersin.org/articles/10.3389/fonc.2021. 681448/full\#supplementary-material 


\section{REFERENCES}

1. Bray F, Ferlay J, Soerjomataram I, Siegel RL, Torre LA, Jemal A. Global Cancer Statistics 2018: GLOBOCAN Estimates of Incidence and Mortality Worldwide for 36 Cancers in 185 Countries. CA Cancer J Clin (2018) 68:394424. doi: $10.3322 /$ caac. 21492

2. Mottet N, Bellmunt J, Bolla M, Briers E, Cumberbatch MG, De Santis M, et al. EAU-ESTRO-SIOG Guidelines on Prostate Cancer. Part 1: Screening, Diagnosis, and Local Treatment With Curative Intent. Eur Urol (2017) 71 (4):618-29. doi: 10.1016/j.eururo.2016.08.003

3. Beckendorf V, Guerif S, Le Prisé E, Cosset JM, Bougnoux A, Chauvet B, et al. 70 Gy Versus 80 Gy in Localized Prostate Cancer: 5-Year Results of GETUG 06 Randomized Trial. Int J Radiat Oncol Biol Phys (2011) 80(4):1056-63. doi: 10.1016/j.ijrobp.2010.03.049

4. Zumsteg ZS, Spratt DE, Romesser PB, Pei X, Zhang Z, Kollmeier M, et al. Anatomical Patterns of Recurrence Following Biochemical Relapse in the Dose Escalation Era of External Beam Radiotherapy for Prostate Cancer. J Urol (2015) 194(6):1624-30. doi: 10.1016/j.juro.2015.06.100

5. Dearnaley DP, Jovic G, Syndikus I, Khoo V, Cowan RA, Graham JD, et al. Escalated-Dose Versus Control-Dose Conformal Radiotherapy for Prostate Cancer: Long-Term Results From the MRC RT01 Randomised Controlled Trial. Lancet Oncol (2014) 15(4):464-73. doi: 10.1016/S1470-2045(14)70040-3

6. Cooperberg MR, Vickers AJ, Broering JM, Carroll PR. Comparative RiskAdjusted Mortality Outcomes After Primary Surgery, Radiotherapy, or Androgen-Deprivation Therapy for Localized Prostate Cancer. Cancer (2010) 116:5226-34. doi: 10.1002/cncr.25456

7. Chatzikonstantinou G, Zamboglou N, Rödel C, Zoga E, Strouthos I, Butt SA, et al. High-Dose-Rate Brachytherapy as Salvage Modality for Locally Recurrent Prostate Cancer After Definitive Radiotherapy: Asystematic Review. Strahlentherapie und Onkol (2017) 193:683-91. doi: 10.1007/ s00066-017-1157-2

8. Zilli T, Benz E, Dipasquale G, Rouzaud M, Miralbell R. Reirradiation of Prostate Cancer Local Failures After Previous Curative Radiation Therapy: Long-Term Outcome and Tolerance. Int J Radiat Oncol Biol Phys (2016) 96:218-322. doi: 10.1016/j.ijrobp.2016.05.024

9. Pasquier D, Martinage G, Janoray G, Rojas DP, Zerini D, Goupy F, et al. Salvage Stereotactic Body Radiation Therapy for Local Prostate Cancer Recurrence After Radiation Therapy: A Retrospective Multicenter Study of the GETUG. Int J Radiat Oncol Biol Phys (2019) 105(4):727-34. doi: 10.1016/ j.ijrobp.2019.07.012

10. Ingrosso G, Becherini C, Lancia A, Caini S, Ost P, Francolini G, et al. Nonsurgical Salvage Local Therapies for Radiorecurrent Prostate Cancer: A Systematic Review and Meta-Analysis. Eur Urol Oncol (2019) 3(2):183-97. doi: 10.1016/j.euo.2018.12.011

11. Chade DC, Eastham J, Graefen M, Hu JC, Karnes RJ, Klotz L, et al. Cancer Control and Functional Outcomes of Salvage Radical Prostatectomy for Radiation-Recurrent Prostate Cancer: A Systematic Review of the Literature. Eur Urol (2012) 61(5):961-71. doi: 10.1016/j.eururo.2012.01.022

12. Moher D, Liberati A, Tetzlaff J, Altman DG, Altman D, Antes G, et al. Preferred Reporting Items for Systematic Reviews and Meta-Analyses: The PRISMA Statement. PloS Med (2009) 6(7):e1000097. doi: 10.1371/ journal.pmed.1000097

13. Moga C, Guo B, Schopflocher D, Harstall C. Development of a Quality Appraisal Tool for Case SeriesStudies Using a Modified Delphi Technique. Edmonton AB: Institute of Health Economics (2012).

14. National Cancer Institute, National Institutes of Health, US Department of Health and Human Services. Common Terminology Criteria for Adverse Events ( CTCAE ) Version 4.0. NIH publication 09-7473. Published May 29, 2009; Revised June 14, 2010. Available at: https://evs.nci.nih.gov/ftp1/ CTCAE/CTCAE 4.03/Archive/CTCAE 4.0_2009-05-29 QuickReference 8. 5x11.pdf (Accessed August 24, 2021).

15. Cox JD, Stetz JA, Pajak TF. Toxicity Criteria of the Radiation Therapy Oncology Group (RTOG) and the European Organization for Research and Treatment of Cancer (EORTC). Int J Radiat Oncol Biol Phys (1995) 31:13416. doi: 10.1016/0360-3016(95)00060-C

16. Peters M, Maenhout M, van der Voort Van Zyp JRN, Moerland MA, Moman MR, Steuten LMG, et al. Focal Salvage Iodine-125 Brachytherapy for Prostate Cancer Recurrences After Primary Radiotherapy: A Retrospective Study
Regarding Toxicity Biochemical Outcome and Quality of Life. Radiother Oncol (2014) 112(1):77-82. doi: 10.1016/j.radonc.2014.06.013

17. Vargas C, Swartz D, Vashi A, Blasser M, Kasraeian A, Cesaretti J, et al. Salvage Brachytherapy for Recurrent Prostate Cancer. Brachytherapy (2014) 13(1):53-8. doi: 10.1016/j.brachy.2013.10.012

18. Smith WH, Cesaretti J, Chin CP, Terk M, Stock RG. Salvage Low Dose Rate Brachytherapy for Prostate Cancer Recurrence Following Definitive External Beam Radiation Therapy. Radiother Oncol (2021) 155:42-7. doi: 10.1016/ j.radonc.2020.10.021

19. Peters M, van der Voort Van Zyp JRN, Moerland MA, Hoekstra CJ, Van De Pol S, Westendorp H, et al. Multivariable Model Development and Internal Validation for Prostate Cancer Specific Survival and Overall Survival After Whole-Gland Salvage Iodine-125 Prostate Brachytherapy. Radiother Oncol (2016) 119(1):104-10. doi: 10.1016/j.radonc.2016.02.002

20. Burri RJ, Stone NN, Unger P, Stock RG. Long-Term Outcome and Toxicity of Salvage Brachytherapy for Local Failure After Initial Radiotherapy for Prostate Cancer. Int J Radiat Oncol Biol Phys (2010) 77(5):1338-44. doi: 10.1016/j.ijrobp.2009.06.061

21. Moman MR, van der Poel HG, Battermann JJ, Moerland MA, van Vulpen M. Treatment Outcome and Toxicity After Salvage 125-I Implantation for Prostate Cancer Recurrences After Primary 125-I Implantation and External Beam Radiotherapy. Brachytherapy (2010) 9(2):119-25. doi: 10.1016/j.brachy.2009.06.007

22. Aaronson DS, Yamasaki I, Gottschalk A, Speight J, Hsu IC, Pickett B, et al. Salvage Permanent Perineal Radioactive-Seed Implantation for Treating Recurrence of Localized Prostate Adenocarcinoma After External Beam Radiotherapy. BJU Int (2009) 104(5):600-4. doi: 10.1111/j.1464-410X.2009. 08445.x

23. Lee HK, Adams MT, Motta J. Salvage Prostate Brachytherapy for Localized Prostate Cancer Failure After External Beam Radiation Therapy. Brachytherapy (2008) 7(1):17-21. doi: 10.1016/j.brachy.2007.11.002

24. Nguyen PL, Chen MH, D'Amico AV, Tempany CM, Steele GS, Albert M, et al. Magnetic Resonance Image-Guided Salvage Brachytherapy After Radiation in Select Men Who Initially Presented With Favorable-Risk Prostate Cancer: A Prospective Phase 2 Study. Cancer (2007) 110(7):1485-192. doi: 10.1002/ cncr.22934

25. Koutrouvelis P, Hendricks F, Lailas N, Gil-Montero G, Sehn J, Khawand N, et al. Salvage Reimplantation in Patient With Local Recurrent Prostate Carcinoma After Brachytherapy With Three Dimensional Computed Tomography-Guided Permanent Pararectal Implant. Technol Cancer Res Treat (2003) 2(4):339-44. doi: 10.1177/153303460300200409

26. Grado GL, Collins JM, Kriegshauser JS, Balch CS, Grado MM, Swanson GP et al. Salvage Brachytherapy for Localized Prostate Cancer After Radiotherapy Failure. Urology (1999) 53(1):2-10. doi: 10.1016/S0090-4295(98)00492-0

27. Crook JM, Zhang P, Pisansky TM, Trabulsi EJ, Amin MB, Bice W, et al. A Prospective Phase 2 Trial of Transperineal Ultrasound-Guided Brachytherapy for Locally Recurrent Prostate Cancer After External Beam Radiation Therapy (NRG Oncology/RTOG-0526). Int J Radiat Oncol Biol Phys (2019) 103 (3):335-43. doi: 10.1016/j.ijrobp.2018.09.039

28. Wojcieszek P, Szlag M, Głowacki G, Cholewka A, Gawkowska-Suwińska M, Kellas-Ślęczka S, et al. Salvage High-Dose-Rate Brachytherapy for Locally Recurrent Prostate Cancer After Primary Radiotherapy Prostate Cancer Salvage Brachytherapy Failure. Radiother Oncol (2016) 119(3):405-10. doi: 10.1016/j.radonc.2016.04.032

29. Yamada Y, Kollmeier MA, Pei X, Kan CC, Cohen GN, Donat SM, et al. A Phase II Study of Salvage High-Dose-Rate Brachytherapy for the Treatment of Locally Recurrent Prostate Cancer After Definitive External Beam Radiotherapy. Brachytherapy (2014) 13(2):111-6. doi: 10.1016/j.brachy.2013.11.005

30. Ware RE, Matthews JP, Hicks RJ, Porceddu S, Hogg A, Rischin D, et al. Usefulness of Fluorine-18 Fluorodeoxyglucose Positron Emission Tomography in Patients With a Residual Structural Abnormality After Difinitive Treatments for Squamous Cell Carcinoma of the Head and Neck. Head Neck (2004) 26(12):1008-17. doi: 10.1002/hed.20097

31. Łyczek J, Kawczyńska MM, Garmol D, Kasprowicz A, Kulik A, Dąbkowski M, et al. HDR Brachytherapy as a Solution in Recurrences of Locally Advanced Prostate Cancer. J Contemp Brachytherapy (2009) 1(2):105-8.

32. Chen CP, Weinberg V, Shinohara K, Roach M, Nash M, Gottschalk A, et al. Salvage HDR Brachytherapy for Recurrent Prostate Cancer After Previous 
Definitive Radiation Therapy: 5-Year Outcomes. Int J Radiat Oncol Biol Phys (2013) 86(2):324-9. doi: 10.1016/j.jijrobp.2013.01.027

33. Lee B, Shinohara K, Weinberg V, Gottschalk AR, Pouliot J, Roach M, et al. Feasibility of High-Dose-Rate Brachytherapy Salvage for Local Prostate Cancer Recurrence After Radiotherapy: The University of California-San Francisco Experience. Int J Radiat Oncol Biol Phys (2007) 67(4):1106-12. doi: 10.1016/j.ijrobp.2006.10.012

34. Slevin F, Hodgson S, Rodda SL, Bownes P, Bottomley D, Adiotomre E, et al. Efficacy and Toxicity Outcomes for Patients Treated With Focal Salvage High Dose Rate Brachytherapy for Locally Recurrent Prostate Cancer. Clin Transl Radiat Oncol (2020) 23:20-6. doi: 10.1016/j.ctro.2020.03.010

35. Kukiełka AM, Hetnał M, Dąbrowski T, Walasek T, Brandys P, Nahajowski D, et al. Salvage Prostate HDR Brachytherapy Combined With Interstitial Hyperthermia for Local Recurrence After Radiation Therapy Failure. Strahlentherapie und Onkol (2014) 190(2):165-70. doi: 10.1007/s00066-0130486-z

36. Chitmanee $\mathrm{P}$, Tsang $\mathrm{Y}$, Tharmalingam $\mathrm{H}$, Hamada $\mathrm{M}$, Alonzi R, Ostler $\mathrm{P}$, et al. Single-Dose Focal Salvage High Dose Rate Brachytherapy for Locally Recurrent Prostate Cancer. Clin Oncol (2020) 32(4):259-65. doi: 10.1016/ j.clon.2019.10.008

37. Jiang P, van der Horst C, Kimmig B, Zinsser F, Poppe B, Luetzen U, et al. Interstitial High-Dose-Rate Brachytherapy as Salvage Treatment for Locally Recurrent Prostate Cancer After Definitive Radiation Therapy: Toxicity and 5-Year Outcome. Brachytherapy (2017) 16(1):186-92. doi: 10.1016/ j.brachy.2016.09.008

38. van Son MJ, Peters M, Moerland MA, Lagendijk JJW, Eppinga WSC, Shah TT, et al. MRI-Guided Ultrafocal Salvage High-Dose-Rate Brachytherapy for Localized Radiorecurrent Prostate Cancer: Updated Results of 50 Patients. Int J Radiat Oncol Biol Phys (2020) 107(1):126-35. doi: 10.1016/j.ijrobp. 2020.01.023

39. Lacy JM, Wilson WA, Bole R, Chen L, Meigooni AS, Rowland RG, et al. Salvage Brachytherapy for Biochemically Recurrent Prostate Cancer Following Primary Brachytherapy. Prostate Cancer (2016) 9561494. doi: 10.1155/2016/ 9561494

40. Kollmeier MA, McBride S, Taggar A, Anderson E, Lin M, Pei X, et al. Salvage Brachytherapy for Recurrent Prostate Cancer After Definitive Radiation Therapy: A Comparison of Low-Dose-Rate and High-Dose-Rate Brachytherapy and the Importance of Prostate-Specific Antigen Doubling Time. Brachytherapy (2017) 16(6):1091-8. doi: 10.1016/j.brachy.2017.07.013

41. Baumann BC, Baumann JC, Christodouleas JP, Soffen E. Salvage of Locally Recurrent Prostate Cancer After External Beam Radiation Using ReducedDose Brachytherapy With Neoadjuvant Plus Adjuvant Androgen Deprivation. Brachytherapy (2017) 16(2):291-8. doi: 10.1016/j.brachy.2016. 12.011

42. Henríquez I, Sancho G, Hervás A, Guix B, Pera J, Gutierrez C, et al. Salvage Brachytherapy in Prostate Local Recurrence After Radiation Therapy: Predicting Factors for Control and Toxicity. Radiat Oncol (2014) 9:102. doi: 10.1186/1748-717X-9-102

43. Henríquez López I, González-San Segundo C, Vegas JO, Gutierrez C, Hervas A, Cabeza Rodriguez MÁ, et al. Salvage Brachytherapy for Locally-Recurrent Prostate Cancer After Radiation Therapy: A Comparison of Efficacy and Toxicity Outcomes With High-Dose Rate and Low-Dose Rate Brachytherapy. Radiother Oncol (2019) 141:156-63. doi: 10.1016/j.radonc.2019.09.006

44. Schönle N, Strnad V, Lotter M, Kreppner S, Fietkau R. Long-Term Results of a Protocol-Based Ultrasound-Guided Salvage Brachytherapy as Re-Irradiation for Local Recurrent Prostate Cancer. Radiother Oncol (2020) 150:201-5. doi: 10.1016/j.radonc.2020.06.031

45. Leroy T, Lacornerie T, Bogart E, Nickers P, Lartigau E, Pasquier D. Salvage Robotic SBRT for Local Prostate Cancer Recurrence After Radiotherapy: Preliminary Results of the Oscar Lambret Center. Radiat Oncol (2017) 12 (1):95. doi: 10.1186/s13014-017-0833-9

46. Bergamin S, Eade T, Kneebone A, Booth J, Hsiao E, Schembri GP, et al. Interim Results of a Prospective Prostate-Specific Membrane AntigenDirected Focal Stereotactic Reirradiation Trial for Locally Recurrent Prostate Cancer. Int J Radiat Oncol Biol Phys (2020) 108(5):1172-8. doi: 10.1016/ j.ijrobp.2020.07.014

47. Fuller D, Wurzer J, Shirazi R, Bridge S, Law J, Crabtree T, et al. Retreatment for Local Recurrence of Prostatic Carcinoma After Prior Therapeutic
Irradiation: Efficacy and Toxicity of HDR-Like SBRT. Int J Radiat Oncol Biol Phys (2020) 106(2):291-9. doi: 10.1016/j.ijrobp.2019.10.014

48. Jereczek-Fossa BA, Patricia Rojas D, Zerini D, Fodor C, Viola A, Fanetti G, et al. Reirradiation for Isolated Local Recurrence of Prostate Cancer: MonoInstitutional Series of 64 Patients Treated With Salvage Stereotactic Body Radiotherapy (SBrT). Br J Radiol (2019) 92(1094):20180494. doi: 10.1259/ bjr.20180494

49. Loi M, Di Cataldo V, Simontacchi G, Detti B, Bonomo P, Masi L, et al. Robotic Stereotactic Retreatment for Biochemical Control in Previously Irradiated Patients Affected by Recurrent Prostate Cancer. Clin Oncol (2018) 30(2):93100. doi: 10.1016/j.clon.2017.11.007

50. D’Agostino GR, Di Brina L, Mancosu P, Franzese C, Iftode C, Franceschini D, et al. Reirradiation of Locally Recurrent Prostate Cancer With Volumetric Modulated Arc Therapy. Int J Radiat Oncol Biol Phys (2019) 104(3):614-21. doi: 10.1016/j.ijrobp.2019.02.041

51. Scher N, Bauduceau O, Bollet M, Lamallem H, Charas T, Garaud P, et al. Stereotactic Prostate Focal Reirradiation Therapy for Local Recurrence: Preliminary Results of Hartmann Oncology Radiotherapy Group. BJR|Open (2019) 1(1):20180027. doi: 10.1259/bjro.20180027

52. Cuccia F, Nicosia L, Mazzola R, Figlia V, Giaj-Levra N, Ricchetti F, et al. Linac-Based SBRT as a Feasible Salvage Option for Local Recurrences in Previously Irradiated Prostate Cancer. Strahlentherapie und Onkol (2020) 196 (7):628-36. doi: 10.1007/s00066-020-01628-6

53. Matrone F, Revelant A, Fanetti G, Polesel J, Chiovati P, Avanzo M, et al. Partial Prostate Re-Irradiation for the Treatment of Isolated Local Recurrence of Prostate Cancer in Patients Previously Treated With Primary External Beam Radiotherapy: Short-Term Results of a Monocentric Study. Neoplasma (2020). 68(1):216-26. doi: 10.4149/neo_2020_200622N651

54. Caroli P, Colangione SP, De Giorgi U, Ghigi G, Celli M, Scarpi E, et al. 68Ga-PSMA-11 PET/CT-Guided Stereotactic Body Radiation Therapy Retreatment in Prostate Cancer Patients With PSA Failure After Salvage Radiotherapy. Biomedicines (2020) 8(12):536. doi: 10.3390/biomedicines 8120536

55. Parker C, Castro E, Fizazi K, Heidenreich A, Ost P, Procopio G, et al. ESMO Guidelines Committee. Electronic address: clinicalguidelines@esmo.org. Prostate Cancer: ESMO Clinical Practice Guidelines for Diagnosis, Treatment and Follow-Up. Ann Oncol (2020) 31(9):1119-34. doi: 10.1016/ j.annonc.2020.06.011

56. Mohler JL, Antonarakis ES, Armstrong AJ, D'Amico AV, Davis BJ, Dorff T, et al. Prostate Cancer, Version 2.2019, NCCN Clinical Practice Guidelines in Oncology. J Natl Compr Cancer Netw (2019) 17(5):479-505. doi: 10.6004/ jnccn.2019.0023

57. Hoskin PJ, Colombo A, Henry A, Niehoff P, Paulsen Hellebust T, Siebert FA, et al. GEC/ESTRO Recommendations on High Dose Rate Afterloading Brachytherapy for Localised Prostate Cancer: An Update. Radiother Oncol (2013) 107(3):325-32. doi: 10.1016/j.radonc.2013.05.002

58. Yamada Y, Rogers L, Demanes DJ, Morton G, Prestidge BR, Pouliot J, et al. American Brachytherapy Society Consensus Guidelines for High-Dose-Rate Prostate Brachytherapy. Brachytherapy (2012) 11(1):20-32. doi: 10.1016/ j.brachy.2011.09.008

59. Golbari NM, Katz AE. Salvage Therapy Options for Local Prostate Cancer Recurrence After Primary Radiotherapy: A Literature Review. Curr Urol Rep (2017) 18(8):63. doi: 10.1007/s11934-017-0709-4

60. Corkum MT, D'Souza DP, Chin J, Boldt G, Mendez LC, Bauman GS. Salvage Reirradiation Using External Beam Radiotherapy for Local Failure in Prostate Cancer: A Systematic Review. Int J Radiat Oncol (2019) 105(1, SUPPLEMENT):E271-2. doi: 10.1016/S0167-8140(19)33355-9

61. Valle LF, Lehrer EJ, Markovic D, Elashoff D, Levin-Epstein R, Karnes RJ, et al. A Systematic Review and Meta-Analysis of Local Salvage Therapies After Radiotherapy for Prostate Cancer (MASTER). Eur Urol (2020) 1(310):1-13. doi: 10.1016/j.eururo.2020.11.010

62. Spratt DE, Scala LM, Folkert M, Voros L, Cohen GN, Happersett L, et al. A Comparative Dosimetric Analysis of Virtual Stereotactic Body Radiotherapy to High-Dose-Rate Monotherapy for Intermediate-Risk Prostate Cancer. Brachytherapy (2013) 12(5):428-33. doi: 10.1016/j.brachy.2013.03.003

63. Tocco BR, Kishan AU, Ma TM, Kerkmeijer LGW, Tree AC. MR-Guided Radiotherapy for Prostate Cancer. Front Oncol (2020) 10:1-11. doi: 10.3389/ fonc.2020.616291 
64. Rose T, Garcia E, Bachand F, Kim D, Petrik D, Halperin R, et al. QOL Comparison of Acute Side Effects From a High Dose Rate vs. Low Dose Rate Prostate Brachytherapy Boost Combined With External Beam Radiotherapy. Brachytherapy (2015) 14:S36. doi: 10.1016/j.brachy.2015.02.245

65. Hathout L, Mahmoud O, Wang Y, Vergalasova I, Barkati M, Després P, et al. A Phase 2 Randomized Pilot Study Comparing High-Dose-Rate Brachytherapy and Low-Dose-Rate Brachytherapy as Monotherapy in Localized Prostate Cancer. Adv Radiat Oncol (2019) 4(4):631-40. doi: 10.1016/j.adro.2019.04.003

66. Rodda S, Tyldesley S, Morris WJ, Keyes M, Halperin R, Pai H, et al. ASCENDE-RT: An Analysis of Treatment-Related Morbidity for a Randomized Trial Comparing a Low-Dose-Rate Brachytherapy Boost With a Dose-Escalated External Beam Boost for High- and Intermediate-Risk Prostate Cancer. Int J Radiat Oncol Biol Phys (2017) 98(2):286-95. doi: 10.1016/j.ijrobp.2017.01.008

67. Hoskin PJ, Rojas AM, Ostler PJ, Bryant L, Lowe GJ. Randomised Trial of External-Beam Radiotherapy Alone or With High-Dose-Rate Brachytherapy for Prostate Cancer: Mature 12-Year Results. Radiother Oncol (2020) 154:2149. doi: 10.1016/j.radonc.2020.09.047

68. Parry MG, Nossiter J, Sujenthiran A, Cowling TE, Patel RN, Morris M, et al. Impact of High-Dose-Rate and Low-Dose-Rate Brachytherapy Boost on Toxicity, Functional and Cancer Outcomes in Patients Receiving External Beam Radiation Therapy for Prostate Cancer: A National Population-Based Study. Int J Radiat Oncol Biol Phys (2021) 109(5):1219-29. doi: 10.1016/ j.ijrobp.2020.11.023

69. Corkum MT, Mendez LC, Chin J, D'Souza D, Boldt RG, Bauman GS. A Novel Salvage Option for Local Failure in Prostate Cancer, Reirradiation Using External Beam or Stereotactic Radiation Therapy: Systematic Review and Meta-Analysis. Adv Radiat Oncol (2020) 5(5):965-77. doi: 10.1016/ j.adro.2020.04.022

70. Jereczek-Fossa BA, Marvaso G, Zaffaroni M, Gugliandolo SG, Zerini D, Corso F, et al. Salvage Stereotactic Body Radiotherapy (SBRT) for Intraprostatic Relapse After Prostate Cancer Radiotherapy: An ESTRO ACROP Delphi Consensus. Cancer Treat Rev (2021) 98:102206. doi: 10.1016/j.ctrv.2021.102206

71. Chen RC, Chang P, Vetter RJ, Lukka H, Stokes WA, Sanda MG, et al. Recommended Patient-Reported Core Set of Symptoms to Measure in Prostate Cancer Treatment Trials. J Natl Cancer Inst (2014) 106(7):dju132. doi: 10.1093/jnci/dju132

72. Skolarus TA, Dunn RL, Sanda MG, Chang P, Greenfield TK, Litwin MS, et al. Minimally Important Difference for the Expanded Prostate Cancer Index Composite Short Form. Urology (2015) 85(1):101-6. doi: 10.1016/ j.urology.2014.08.044

73. Kaljouw E, Pieters BR, Kovács G, Hoskin PJ. A Delphi Consensus Study on Salvage Brachytherapy for Prostate Cancer Relapse After Radiotherapy, A Uro-GEC Study. Radiother Oncol (2016) 118:122-30. doi: 10.1016/j.radonc.2015.10.021

74. Crook J, Malone S, Perry G, Bahadur Y, Robertson S, Abdolell M. Postradiotherapy Prostate Biopsies: What Do They Really Mean? Results for 498 Patients. Int J Radiat Oncol Biol Phys (2000) 28(2):355-67. doi: 10.1016/S0360-3016(00)00637-4

75. Murgic J, Morton G, Loblaw A, D’Alimonte L, Ravi A, Wronski M, et al. Focal Salvage High Dose-Rate Brachytherapy for Locally Recurrent Prostate Cancer After Primary Radiation Therapy Failure: Results From a Prospective Clinical
Trial. Int J Radiat Oncol Biol Phys (2018) 102(3):561-7. doi: 10.1016/ j.ijrobp.2018.06.039

76. De Visschere PJL, Standaert C, Fütterer JJ, Villeirs GM, Panebianco V, Walz J, et al. A Systematic Review on the Role of Imaging in Early Recurrent Prostate Cancer. Eur Urol Oncol (2019) 2(1):47-76. doi: 10.1016/j.euo.2018.09.010

77. Hövels AM, Heesakkers RAM, Adang EM, Jager GJ, Strum S, Hoogeveen YL, et al. The Diagnostic Accuracy of CT and MRI in the Staging of Pelvic Lymph Nodes in Patients With Prostate Cancer: A Meta-Analysis. Clin Radiol (2008) 63(4):387-95. doi: 10.1016/j.crad.2007.05.022

78. Mertan FV, Greer MD, Borofsky S, Kabakus IM, Merino MJ, Wood BJ, et al. Multiparametric Magnetic Resonance Imaging of Recurrent Prostate Cancer. Top Magn Reson Imaging (2016) 25:139-47. doi: 10.1097/RMR.0000000000000088

79. Afshar-Oromieh A, Avtzi E, Giesel FL, Holland-Letz T, Linhart HG, Eder M, et al. The Diagnostic Value of PET/CT Imaging With the 68Ga-Labelled PSMA Ligand HBED-CC in the Diagnosis of Recurrent Prostate Cancer. Eur J Nucl Med Mol Imaging (2015) 42(2):197-209. doi: 10.1007/s00259-014-2949-6

80. Hruby G, Eade T, Kneebone A, Emmett L, Guo L, Ho B, et al. Delineating Biochemical Failure With 68Ga-PSMA-PET Following Definitive External Beam Radiation Treatment for Prostate Cancer. Radiother Oncol (2017) 122 (1):99-102. doi: 10.1016/j.radonc.2016.11.023

81. Hofman MS, Lawrentschuk N, Francis RJ, Tang C, Vela I, Thomas P, et al. Prostate-Specific Membrane Antigen PET-CT in Patients With High-Risk Prostate Cancer Before Curative-Intent Surgery or Radiotherapy (proPSMA): A Prospective, Randomised, Multicentre Study. Lancet (2020) 395 (10231):1208-16. doi: 10.1016/S0140-6736(20)30314-7

82. Slevin F, Beasley M, Cross W, Scarsbrook A, Murray L, Henry A. Patterns of Lymph Node Failure in Patients With Recurrent Prostate Cancer Postradical Prostatectomy and Implications for Salvage Therapies. Adv Radiat Oncol (2020) 5(6):1126-40. doi: 10.1016/j.adro.2020.07.009

Conflict of Interest: AH served as a guest editor for Frontiers in Oncology.

The remaining authors declare that the research was conducted in the absence of any commercial or financial relationships that could be construed as a potential conflict of interest.

The reviewer NJ declared a past collaboration with the authors $\mathrm{AC}, \mathrm{PH}$ to the handling editor.

Publisher's Note: All claims expressed in this article are solely those of the authors and do not necessarily represent those of their affiliated organizations, or those of the publisher, the editors and the reviewers. Any product that may be evaluated in this article, or claim that may be made by its manufacturer, is not guaranteed or endorsed by the publisher.

Copyright (c) 2021 Zhong, Slevin, Scarsbrook, Serra, Choudhury, Hoskin, Brown and Henry. This is an open-access article distributed under the terms of the Creative Commons Attribution License (CC BY). The use, distribution or reproduction in other forums is permitted, provided the original author(s) and the copyright owner(s) are credited and that the original publication in this journal is cited, in accordance with accepted academic practice. No use, distribution or reproduction is permitted which does not comply with these terms. 